\title{
Reduction of Couplings: Finite Unified Theories and the reduced MSSM
}

\author{
Sven Heinemeyer \\ Instituto de Física Teórica, Universidad Autónoma de Madrid, Cantoblanco, \\ 28049 Madrid, Spain \\ Campus of International Excellence UAM+CSIC, Cantoblanco, 28049 Madrid, Spain \\ Instituto de Física de Cantabria (CSIC-UC), E-39005 Santander, Spain \\ E-mail: Sven.Heinemeyerecern.ch

\section{Myriam Mondragón*} \\ Instituto de Física, Universidad Nacional Autónoma de México, A.P. 20-364, México 01000 \\ E-mail: myriamefisica.unam.mx
}

\section{Gregory Patellis}

Physics Department, Nat. Technical University, 15780 Zografou, Athens, Greece

E-mail: patellis@central.ntua.gr

\section{Nicholas Tracas}

Physics Department, Nat. Technical University, 15780 Zografou, Athens, Greece

E-mail: ntracecentral.ntua.gr

\section{George Zoupanos}

Physics Department, Nat. Technical University, 15780 Zografou, Athens, Greece Max-Planck Institut für Physik, Föhringer Ring 6, D-80805 München, Germany

E-mail: George.Zoupanosecern.ch

The principle of reduction of couplings, combined with $N=1$ supersymmetry, can lead to realistic quantum field theories, where the gauge and Yukawa sectors are related. It is the basis to find allloop Finite Unified Theories (FUTs), where the $\beta$-function vanishes to all loops in perturbation theory. It can also be applied to the Minimal Supersymmetric Standard Model (MSSM), leading to an important reduction of the number of parameters. We present results for the $N=1 S U(5)$ FUT, the $N=1 S U(3) \otimes S U(3) \otimes S U(3)$ FUT and the reduced MSSM. These models lead to successful predictions for the masses of the third generation of quarks and the Higgs boson. They also predict a heavy supersymmetric spectrum, consistent with the non-observation of supersymmetry so far. The FCC-hh will be able to fully test the predicted spectrum.

Corfu Summer Institute 2018 'Workshops on Elementary Particle Physics and Gravity'

31 August - 29 September 2018

Corfu, Greece

IFT-UAM/CSIC-19-093

\footnotetext{
${ }^{*}$ Speaker.
} 


\section{Introduction}

The particle discovered at the LHC in 2012 [1,2] is, within uncertainties, compatible with predictions for the Higgs boson of the Standard Model (SM) [3]. Therefore the SM can be considered as a complete theory of the low energy of Particle Physics. However problems such as the hierarchy, the neutrino masses, the dark matter, the over twenty free parameters of the SM suggest the existence of a more fundamental theory at higher scales. Furthermore, the hierarchy problem, the neutrino masses, the dark matter, the over twenty free parameters of the SM ask for a more fundamental theory.

Therefore, one of the main aims of this fundamental theory is to relate these free parameters, or, in other words, to reduce the number of these parameters. This reduction is usually based on the introduction of a larger symmetry, rendering the theory more predictive, such as Grand Unified Theories (GUTs) [4-10]. One example is the minimal $S U(5)$, where the number of gauge couplings is reduced to one due to unification. Data from LEP [11] suggested that a $N=1$ global Supersymmetry (SUSY) $[9,10]$ broken in the TeV scale is necessary to be introduced for the unification to be viable. Relations among the Yukawa couplings are also suggested in GUTs. The $S U(5)$, for example, predicts the ratio of tau mass to bottom mass $M_{\tau} / M_{b}$ [12] in the SM. However, GUTs based on larger gauge groups introduce new degrees of freedom and loose predictivity due to the different ways of breaking the larger symmetry.

One way to reduce further the couplings of a GUT is to relate its Yukawa and gauge sectors (Gauge-Yukawa Unification - GYU) [13-15]. Unfortunately the interesting possibility that $N=2$ SUSY [16] could play this role is highly limited, since it predicts the existence of light mirror fermions. Phenomenological problems also appear in composite models and superstring theories.

Another approach is to search for all-loop Renormalization Group Invariant (RGI) relations among Yukawa and gauge couplings $[17,18]$, which hold from the unification scale up to the Planck scale [13-15,19-24]. With this approach GYU is possible. Remarkably, RGI expressions that guarantee finiteness to all orders in perturbation theory can be found, too, assuming, among others, finiteness at one loop in $N=1$ gauge theories [25-27].

The above approach needs SUSY as an essential ingredient. Moreover, the SUSY breaking sector has to be treated in a similar way, since it introduces several new parameters in the theory. In fact, the RGI relation searches have been extended to the Soft SUSY Breaking (SSB) sector [24, 28-30] relating parameters of mass dimension one and two.

Reduction of couplings in $N=1$ SUSY theories has led to very interesting phenomenological developments [31]. In past works, a "universal" set of soft scalar masses was assumed in the soft breaking sector, given that: (1) they are part of constraints that preserve two-loop finiteness [32,33]; (2) they are two-loop RGI in more general SUSY gauge theories, subject to the condition known as $P=1 / 3 Q$ [28]; and (3) they appear in dilaton-dominated SUSY breaking superstring scenarios [34-36]. However, phenomenological problems occur, all due to the restrictive nature of the "universality" assumption for the soft scalar masses. For instance: (a) in Finite Unified Theories (FUTs) universality predicts that the LSP is charged, namely the superpartner of the $\tau$ lepton $\tilde{\tau}$; (b) the Minimal Supersymmetric Standard Model (MSSM) with universal soft scalar masses is incompatible with radiative electroweak symmetry breaking; and worst of all, (c) the universal condition leads to charge and/or color breaking minima deeper than the standard vacuum [37]. There 
have been attempts, consequently, to relax this condition without loosing its attractive features. First, there is an interesting observation in $N=1 \mathrm{GYU}$ theories: there exists an RGI sum rule for the soft scalar masses at lower orders; at one loop for the non-finite case [38] and at two loops for the finite case [39]. The sum rule overcomes the above unpleasant phenomenological consequences. Moreover, the sum rule for the soft scalar masses is RGI to all orders [40] for both the general and the finite case. Finally, the exact $\beta$-functions for soft scalar masses in the NovikovShifman-Vainshtein-Zakharov (NSVZ) scheme [41-43] for the softly broken SUSY QCD have been obtained [40]. The use of RGI both in the dimensionful and the dimensionless sector together with the sum rule allows the construction of realistic and predictive $N=1$ all-loop finite $S U(5)$ SUSY models with interesting predictions, as shown in [30,44-48].

\section{Theoretical Basis}

In this section we will describe the principle of reduction of couplings. Any RGI relation among parameters (which does not depend on the renormalization scale $\mu$ explicitly) can be expressed in the implicit form $\Phi\left(g_{1}, \cdots, g_{A}\right)=$ const., which should satisfy the partial differential equation (PDE):

$$
\mu \frac{d \Phi}{d \mu}=\vec{\nabla} \cdot \vec{\beta}=\sum_{a=1}^{A} \beta_{a} \frac{\partial \Phi}{\partial g_{a}}=0,
$$

where $\beta_{a}$ is the $\beta$-function of $g_{a}$. This PDE is equivalent to a set of ordinary differential equations (ODEs), the so-called reduction equations (REs) [17, 18,49]

$$
\beta_{g} \frac{d g_{a}}{d g}=\beta_{a}, a=1, \cdots, A
$$

where $g$ and $\beta_{g}$ are the primary coupling and its $\beta$-function. The counting on $a$ does not include $g$. Since maximally $(A-1)$ independent RGI "constraints" can be imposed by the $\Phi_{a}$ s, all parameters can in principle be expressed in terms of a single parameter $g$. A closer look at the set of Eq. (2.2), however, reveals that their general solutions contain as many integration constants as the number of equations themselves. That means that we have just traded an integration constant for each ordinary renormalized coupling. Consequently, the general solutions cannot be considered as reduced ones. The crucial requirement in the search for RGI relations is to demand power series solutions to the REs,

$$
g_{a}=\sum_{n} \rho_{a}^{(n)} g^{2 n+1}
$$

These solutions preserve perturbative renormalizability. This ansatz fixes the corresponding integration constant in each of the REs and picks up a special solution out of each general one. It is worth noting that the uniqueness of such power series solutions can be decided at one-loop level $[17,18,49]$.

Coupling unification, as described in this section, is very attractive, since the "completely reduced" theory contains only one independent parameter. However, for some models it can be unrealistic. Therefore, we often impose fewer RGI constraints. This is the idea of partial reduction $[50,51]$. 


\section{Finiteness in $N=1$ Supersymmetric Gauge Theories}

\subsection{Finiteness}

The superpotential of a chiral, anomaly-free, $N=1$ globally supersymmetric gauge theory based on a group $\mathrm{G}$ with gauge coupling constant $g$ is given by

$$
W=\frac{1}{2} m_{i j} \phi_{i} \phi_{j}+\frac{1}{6} C_{i j k} \phi_{i} \phi_{j} \phi_{k},
$$

where $m_{i j}$ and $C_{i j k}$ are gauge invariant tensors and the matter field $\phi_{i}$ transforms according to the irreducible representation $R_{i}$ of the gauge group $G$. Then, the renormalization constants associated with the superpotential (3.1), assuming that supersymmetry is exact, are:

$$
\begin{aligned}
\phi_{i}^{0} & =\left(Z_{i}^{j}\right)^{(1 / 2)} \phi_{j}, \\
m_{i j}^{0} & =Z_{i j}^{i^{\prime} j^{\prime}} m_{i^{\prime} j^{\prime}}, \\
C_{i j k}^{0} & =Z_{i j k}^{i^{\prime} j^{\prime} k^{\prime}} C_{i^{\prime} j^{\prime} k^{\prime}} .
\end{aligned}
$$

By virtue of the $N=1$ non-renormalization theorem [54-56] there are no mass and cubicinteraction-term infinities, therefore:

$$
\begin{aligned}
Z_{i j k}^{i^{\prime} j^{\prime} k^{\prime}} Z_{i^{\prime}}^{1 / 2 i^{\prime \prime}} Z_{j^{\prime}}^{1 / 2 j^{\prime \prime}} Z_{k^{\prime}}^{1 / 2 k^{\prime \prime}} & =\delta_{(i}^{i^{\prime \prime}} \delta_{j}^{j^{\prime \prime}} \delta_{k)}^{k^{\prime \prime}}, \\
Z_{i j}^{i^{\prime} j^{\prime}} Z_{i^{\prime}}^{1 / 2 i^{\prime \prime}} Z_{j^{\prime}}^{1 / 2 j^{\prime \prime}} & =\delta_{(i}^{i^{\prime \prime}} \delta_{j)}^{j^{\prime \prime}} .
\end{aligned}
$$

Thus, the only surviving infinities are the wave-function renormalization constants $Z_{i}^{j}$, i.e. one infinity for each field. The one-loop $\beta$-function of the gauge coupling $g$ is given by [57]:

$$
\beta_{g}^{(1)}=\frac{d g}{d t}=\frac{g^{3}}{16 \pi^{2}}\left[\sum_{i} l\left(R_{i}\right)-3 C_{2}(G)\right]
$$

where $l\left(R_{i}\right)$ is the Dynkin index of $R_{i}$ and $C_{2}(G)$ is the quadratic Casimir operator of the adjoint representation of the gauge group $G$. The $\beta$-functions of $C_{i j k}$, due to the non-renormalization theorem, are proportional to the anomalous dimensions $\gamma_{i j}$ of the matter fields $\phi_{i}$ :

$$
\beta_{i j k}=\frac{d C_{i j k}}{d t}=C_{i j l} \gamma_{k}^{l}+C_{i k l} \gamma_{j}^{l}+C_{j k l} \gamma_{i}^{l}
$$

where at one-loop $\gamma_{i j}$ is given by [57]:

$$
\gamma_{j}^{i(1)}=\frac{1}{32 \pi^{2}}\left[C^{i k l} C_{j k l}-2 g^{2} C_{2}(R) \delta_{j}^{i}\right],
$$

where $C^{i j k}=C_{i j k}^{*}$. We may assume that $C_{i j k}$ are real (so $C_{i j k}^{2}$ are always positive numbers).

One can see from Equations (3.6) and (3.8) that all the one-loop $\beta$-functions of the theory vanish if $\beta_{g}^{(1)}$ and $\gamma_{i j}^{(1)}$ vanish:

$$
\begin{gathered}
\sum_{i} \ell\left(R_{i}\right)=3 C_{2}(G), \\
C^{i k l} C_{j k l}=2 \delta_{j}^{i} g^{2} C_{2}\left(R_{i}\right) .
\end{gathered}
$$


The conditions for finiteness for $N=1$ theories with $S U(N)$ gauge structure are discussed in [58] and the analysis for the anomaly-free and no-charge renormalization requirements for these theories is found in [59]. An interesting result is that conditions (3.9) and (3.10) are also necessary and sufficient for finiteness at the two-loop level [57, 60-63].

In the case that supersymmetry is broken by the inclusion of soft terms, the requirement of finiteness in the one-loop soft breaking terms imposes further constraints [32]. In addition, the set of conditions that are sufficient for one-loop finiteness of the soft terms renders the soft sector two-loop finite as well [33].

The one-loop and two-loop finiteness conditions (3.9) and (3.10) restrict considerably the possible choices of the irreducible representations (irreps) $R_{i}$ for a group $G$, as well as Yukawa couplings in the superpotential (3.1). Note that these finiteness conditions cannot be applied to the Minimal Supersymmetric Standard Model (MSSM), since the $U(1)$ gauge group is incompatible with condition (3.9) due to $C_{2}[U(1)]=0$. This leads to the expectation that finiteness will be attained at GUT level only, the MSSM being just the corresponding low-energy effective theory.

Another important consequence of one- and two-loop finiteness is that supersymmetry can only be broken softly. Indeed, gauge singlets are unacceptable, F-type spontaneous symmetry breaking terms [64] are incompatible with finiteness, as well as D-type [65] spontaneous breaking, as it requires a $U(1)$ gauge group.

A natural question to ask is what happens at higher orders. The answer can be found in a theorem $[26,66]$ that states the necessary and sufficient conditions to achieve all-loop finiteness. Before we discuss the theorem, some introductory remarks are in order. The finiteness conditions impose relations between gauge and Yukawa couplings. However, it is trivial to require such relations that render the couplings mutually dependent at a given renormalization point. What is non-trivial is to guarantee that relations leading to reduction of couplings hold at any renormalization point. As we already know, the necessary and sufficient condition for this to happen is to require that such relations are solutions to the REs

$$
\beta_{g} \frac{d C_{i j k}}{d g}=\beta_{i j k}
$$

and hold at all orders. It is remarkable that the existence of all-loop power series solutions to (3.11) can be decided at one-loop level, as it was already mentioned.

Let us now turn to the all-order finiteness theorem $[26,66]$, which states the conditions under which an $N=1$ SUSY gauge theory can become finite to all orders in the sense of vanishing $\beta$ functions, that is of physical scale invariance. It is based on (a) the structure of the supercurrent in $N=1$ SUSY gauge theory [67-69] and on (b) the non-renormalization properties of $N=1$ chiral anomalies $[25,26,66,70,71]$. Details on the proof can be found in $[26,66]$ and further discussion in Refs. [25, 27, 70-72].

Theorem: Consider an N=1 SUSY Yang-Mills theory, with the simple gauge group. If the following conditions are satisfied:

1. There is no gauge anomaly. 
2. The gauge $\beta$-function vanishes at one loop:

$$
\beta_{g}^{(1)}=0=\sum_{i} l\left(R_{i}\right)-3 C_{2}(G) .
$$

3. There exist solutions of the form:

$$
C_{i j k}=\rho_{i j k} g, \quad \rho_{i j k} \in \mathbb{C}
$$

to the conditions of vanishing one-loop matter fields' anomalous dimensions:

$$
\begin{aligned}
& \gamma_{j}^{i(1)}=0 \\
& =\frac{1}{32 \pi^{2}}\left[C^{i k l} C_{j k l}-2 g^{2} C_{2}(R) \delta_{j}^{i}\right] .
\end{aligned}
$$

4. These solutions are isolated and non-degenerate when considered as solutions of vanishing one-loop Yukawa $\beta$-functions:

$$
\beta_{i j k}=0
$$

Then, each of the solutions (3.13) can be uniquely extended to a formal power series in $g$, and the associated super Yang-Mills models depend on the single coupling constant $g$ with a $\beta$ function, which vanishes at all orders.

It is important to note that the requirement of isolated and non-degenerate solutions guarantees the existence of a unique formal power series solution to the reduction equations. Thus, we see that finiteness and reduction of couplings are intimately related. Also, we should note that one cannot extend the validity of a similar theorem in non-SUSY theories.

\subsection{Partial Reduction}

For our purposes, it is convenient to work with the square of the couplings and to arrange $C_{i j k}$ in such a way that they are covered by a single index $i(i=1, \cdots, n)$ :

$$
\alpha=\frac{g^{2}}{4 \pi}, \alpha_{i}=\frac{g_{i}^{2}}{4 \pi} .
$$

The evolution equations of $\alpha$ 's in perturbation theory then take the form

$$
\begin{aligned}
& \frac{d \alpha}{d t}=\beta=-\beta^{(1)} \alpha^{2}+\cdots \\
& \frac{d \alpha_{i}}{d t}=\beta_{i}=-\beta_{i}^{(1)} \alpha_{i} \alpha+\sum_{j, k} \beta_{i, j k}^{(1)} \alpha_{j} \alpha_{k}+\cdots
\end{aligned}
$$

where $\cdots$ denotes the contributions from higher orders, and $\beta_{i, j k}^{(1)}=\beta_{i, k j}^{(1)}$.

Given the set of the evolution equations (3.17), we investigate the asymptotic properties, as follows. First we define [17, 49, 73-75]

$$
\tilde{\alpha}_{i} \equiv \frac{\alpha_{i}}{\alpha}, i=1, \cdots, n,
$$


and derive from Eq. (3.17)

$$
\begin{aligned}
\alpha \frac{d \tilde{\alpha}_{i}}{d \alpha} & =-\tilde{\alpha}_{i}+\frac{\beta_{i}}{\beta}=\left(-1+\frac{\beta_{i}^{(1)}}{\beta^{(1)}}\right) \tilde{\alpha}_{i} \\
& -\sum_{j, k} \frac{\beta_{i, j k}^{(1)}}{\beta^{(1)}} \tilde{\alpha}_{j} \tilde{\alpha}_{k}+\sum_{r=2}\left(\frac{\alpha}{\pi}\right)^{r-1} \tilde{\beta}_{i}^{(r)}(\tilde{\alpha}),
\end{aligned}
$$

where $\tilde{\beta}_{i}^{(r)}(\tilde{\alpha})(r=2, \cdots)$ are power series of $\tilde{\alpha}$ 's and can be computed from the $r$-th loop $\beta$ functions. Next we search for fixed points $\rho_{i}$ of Eq. (3.18) at $\alpha=0$. To this end, we have to solve

$$
\left(-1+\frac{\beta_{i}^{(1)}}{\beta^{(1)}}\right) \rho_{i}-\sum_{j, k} \frac{\beta_{i, j k}^{(1)}}{\beta^{(1)}} \rho_{j} \rho_{k}=0,
$$

and assume that the fixed points have the form

$$
\rho_{i}=0 \text { for } i=1, \cdots, n^{\prime} ; \rho_{i}>0 \text { for } i=n^{\prime}+1, \cdots, n .
$$

We then regard $\tilde{\alpha}_{i}$ with $i \leq n^{\prime}$ as small perturbations to the undisturbed system which is defined by setting $\tilde{\alpha}_{i}$ with $i \leq n^{\prime}$ equal to zero. As we have seen, it is possible to verify at the one-loop level $[17,18,49,73]$ the existence of the unique power series solution

$$
\tilde{\alpha}_{i}=\rho_{i}+\sum_{r=2} \rho_{i}^{(r)} \alpha^{r-1}, i=n^{\prime}+1, \cdots, n
$$

of the reduction equations (3.19) to all orders in the undisturbed system. These are RGI relations among couplings and keep formally perturbative renormalizability of the undisturbed system. So in the undisturbed system there is only one independent coupling, the primary coupling $\alpha$.

The small perturbations caused by nonvanishing $\tilde{\alpha}_{i}$ with $i \leq n^{\prime}$ enter in such a way that the reduced couplings, i.e. $\tilde{\alpha}_{i}$ with $i>n^{\prime}$, become functions not only of $\alpha$ but also of $\tilde{\alpha}_{i}$ with $i \leq n^{\prime}$. It turned out that, to investigate such partially reduced systems, it is most convenient to work with the partial differential equations

$$
\begin{gathered}
\left\{\tilde{\beta} \frac{\partial}{\partial \alpha}+\sum_{a=1}^{n^{\prime}} \tilde{\beta}_{a} \frac{\partial}{\partial \tilde{\alpha}_{a}}\right\} \tilde{\alpha}_{i}(\alpha, \tilde{\alpha})=\tilde{\beta}_{i}(\alpha, \tilde{\alpha}) \\
\tilde{\beta}_{i(a)}=\frac{\beta_{i(a)}}{\alpha^{2}}-\frac{\beta}{\alpha^{2}} \tilde{\alpha}_{i(a)}, \quad \tilde{\beta} \equiv \frac{\beta}{\alpha},
\end{gathered}
$$

which are equivalent to the reduction equations (3.19), where we let $a, b$ run from 1 to $n^{\prime}$ and $i, j$ from $n^{\prime}+1$ to $n$ in order to avoid confusion. We then look for solutions of the form

$$
\tilde{\alpha}_{i}=\rho_{i}+\sum_{r=2}\left(\frac{\alpha}{\pi}\right)^{r-1} f_{i}^{(r)}\left(\tilde{\alpha}_{a}\right), i=n^{\prime}+1, \cdots, n,
$$

where $f_{i}^{(r)}\left(\tilde{\alpha}_{a}\right)$ are supposed to be power series of $\tilde{\alpha}_{a}$. This particular type of solution can be motivated by requiring that in the limit of vanishing perturbations we obtain the undisturbed solutions (3.22) [51,76]. Again, it is possible to obtain the sufficient conditions for the uniqueness of $f_{i}^{(r)}$ in terms of the lowest order coefficients. 


\section{The SSB Sector of Reduced $N=1$ SUSY and Finite Theories}

The method of reducing the dimensionless couplings has been extended [24] to the soft supersymmetry breaking (SSB) dimensionful parameters of $N=1$ SUSY theories. In addition, it was found [38] that SSB scalar masses in GYU models satisfy an RGI sum rule.

Consider the superpotential given by (3.1) along with the Lagrangian for SSB terms:

$$
\begin{aligned}
-\mathscr{L}_{\mathrm{SSB}} & =\frac{1}{6} h^{i j k} \phi_{i} \phi_{j} \phi_{k}+\frac{1}{2} b^{i j} \phi_{i} \phi_{j} \\
& +\frac{1}{2}\left(m^{2}\right)_{i}^{j} \phi^{* i} \phi_{j}+\frac{1}{2} M \lambda \lambda+\text { h.c. },
\end{aligned}
$$

where $\phi_{i}$ are the scalar parts of chiral superfields $\Phi_{i}, \lambda$ are the gauginos and $M$ their unified mass.

We assume that the REs admit power series solutions:

$$
C^{i j k}=g \sum_{n} \rho_{(n)}^{i j k} g^{2 n}
$$

In the case of finite theories, we further assume that the gauge group is a simple group and the one-loop gauge $\beta$-function vanishes. According to the finiteness theorem Refs. $[26,66]$ and the assumption given in (4.1), the theory is then all-order finite, if, among others, the one-loop anomalous dimensions $\gamma_{i}^{j(1)}$ vanish. The one-loop and two-loop finiteness for $h^{i j k}$ can be achieved by [33]:

$$
h^{i j k}=-M C^{i j k}+\cdots=-M \rho_{(0)}^{i j k} g+O\left(g^{5}\right),
$$

where ... stand for higher order terms.

In order to obtain the two-loop sum rule for soft scalar masses, we will assume that the lowest order coefficients $\rho_{(0)}^{i j k}$ and also $\left(m^{2}\right)_{j}^{i}$ satisfy the diagonality relations:

$$
\rho_{i p q(0)} \rho_{(0)}^{j p q} \propto \delta_{i}^{j} \quad \text { for all } p \text { and } q \text { and } \quad\left(m^{2}\right)_{j}^{i}=m_{j}^{2} \delta_{j}^{i}
$$

Then the following soft scalar-mass sum rule is found $[15,39,81]$ :

$$
\left(m_{i}^{2}+m_{j}^{2}+m_{k}^{2}\right) / M M^{\dagger}=1+\frac{g^{2}}{16 \pi^{2}} \Delta^{(2)}+O\left(g^{4}\right)
$$

for $\mathrm{i}, \mathrm{j}$, k with $\rho_{(0)}^{i j k} \neq 0 . \Delta^{(2)}$ is the two-loop correction

$$
\Delta^{(2)}=-2 \sum_{l}\left[\left(m_{l}^{2} / M M^{\dagger}\right)-(1 / 3)\right] T\left(R_{l}\right),
$$

which vanishes for the universal choice in accordance with the previous findings of Ref. [33]. 
Using the spurion technique [56, 82-85], one can find the following all-loop relations among $\beta$-functions [86-91]:

$$
\begin{aligned}
\beta_{M} & =2 \mathscr{O}\left(\frac{\beta_{g}}{g}\right), \\
\beta_{h}^{i j k} & =\gamma^{i}{ }_{l} h^{l j k}+\gamma^{j} h^{i l k}+\gamma^{k} h^{i j l} \\
& -2 \gamma_{1 l}^{i} C^{l j k}-2 \gamma_{1}^{j} C^{i l k}-2 \gamma_{1 l}^{k} C^{i j l}, \\
\left(\beta_{m^{2}}\right)^{i}{ }_{j} & =\left[\Delta+X \frac{\partial}{\partial g}\right] \gamma^{i}, \\
\mathscr{O} & =\left(M g^{2} \frac{\partial}{\partial g^{2}}-h^{l m n} \frac{\partial}{\partial C^{l m n}}\right), \\
\Delta & =2 \mathscr{O} \mathscr{O}^{*}+2|M|^{2} g^{2} \frac{\partial}{\partial g^{2}}+\tilde{C}_{l m n} \frac{\partial}{\partial C_{l m n}}+\tilde{C}^{l m n} \frac{\partial}{\partial C^{l m n}},
\end{aligned}
$$

where $\left(\gamma_{1}\right)^{i}{ }_{j}=\mathscr{O} \gamma_{j}^{i}, C_{l m n}=\left(C^{l m n}\right)^{*}$, and:

$$
\tilde{C}^{i j k}=\left(m^{2}\right)^{i}{ }_{l} C^{l j k}+\left(m^{2}\right)^{j}{ }_{l} C^{i l k}+\left(m^{2}\right)^{k}{ }_{l} C^{i j l} .
$$

The key point in the strategy of refs. $[40,90,91]$ is the assumption that the differential operators $\mathscr{O}$ and $\Delta$ given in eqs. (4.9) and (4.10) become total derivative operators on certain RG invariant surfaces. Therefore the the beta functions of the exact theory are directly related to those of the soft supersymmetry breaking terms. Maybe the most interesting result appears in the case of finite theories in which the property of vanishing beta-functions of the exact theory is transferred to the beta functions of the softly broken sector and therefore the whole theory becomes finite.

In particular, and using the all-loop gauge $\beta$-function of Novikov et al. [41-43] given by:

$$
\beta_{g}^{\mathrm{NSVZ}}=\frac{g^{3}}{16 \pi^{2}}\left[\frac{\sum_{l} T\left(R_{l}\right)\left(1-\gamma_{l} / 2\right)-3 C(G)}{1-g^{2} C(G) / 8 \pi^{2}}\right],
$$

the all-loop RGI sum rule [40] has been found:

$$
\begin{aligned}
m_{i}^{2}+m_{j}^{2}+m_{k}^{2} & =|M|^{2}\left\{\frac{1}{1-g^{2} C(G) /\left(8 \pi^{2}\right)} \frac{d \ln C^{i j k}}{d \ln g}+\frac{1}{2} \frac{d^{2} \ln C^{i j k}}{d(\ln g)^{2}}\right\} \\
& +\sum_{l} \frac{m_{l}^{2} T\left(R_{l}\right)}{C(G)-8 \pi^{2} / g^{2}} \frac{d \ln C^{i j k}}{d \ln g}
\end{aligned}
$$

Also, the exact $\beta$-function for $m^{2}$ in the NSVZ scheme has been obtained [40] for the first time:

$$
\begin{aligned}
\beta_{m_{i}^{2}}^{\mathrm{NSVZ}} & =\left[|M|^{2}\left\{\frac{1}{1-g^{2} C(G) /\left(8 \pi^{2}\right)} \frac{d}{d \ln g}+\frac{1}{2} \frac{d^{2}}{d(\ln g)^{2}}\right\}\right. \\
& \left.+\sum_{l} \frac{m_{l}^{2} T\left(R_{l}\right)}{C(G)-8 \pi^{2} / g^{2}} \frac{d}{d \ln g}\right] \gamma_{i}^{\mathrm{NSVZ}}
\end{aligned}
$$

Surprisingly, the all-loop result (4.13) coincides with the superstring result for the finite case in a certain class of orbifold models [39] if $d \ln C^{i j k} / d \ln g=1$. 
Finally, it is important to emphasize that the sum rule holds always, to the extend that there is reduction of couplings. A consequence from the reduction of dimensionful parameters is that in some cases it is possible to have exact relations among the soft scalar masses and a mass-dimension one parameter (which could be the gaugino unified mass). This option is not phenomenologically viable for the case of Finite Unified Theories, though.

\section{Reduction of Couplings in Phenomenologically Viable Models}

\subsection{A Successful Finite Unified Theory}

In this section we will review an all-loop FUT with $S U(5)$ as the gauge group, where the reduction of couplings has been applied to the third fermionic generation [19]. This model was selected on the basis of agreement with known experimental data at the time [45]. It predicted the light Higgs boson mass to be in the range $121-126 \mathrm{GeV}$ four and a half years before the discovery. The particle content of the model we will study, which we denote $S U(5)$-FUT, consists of three $(\overline{\mathbf{5}}+\mathbf{1 0})$ supermultiplets needed for each of the three generations of quarks and leptons, four $(\overline{\mathbf{5}}+\mathbf{5})$ and one $\mathbf{2 4}$ considered as Higgs supermultiplets. When the gauge group of the FUT is broken, the theory is no longer finite and we are left with the MSSM [14,19-23].

A predictive GYU all-loop finite $S U(5)$ model, in addition to the above-mentioned requirements, should have the following properties:

1. One-loop anomalous dimensions are diagonal: $\gamma_{i}^{(1) j} \propto \delta_{i}^{j}$.

2. Three fermion generations in the irreducible representations $\overline{\mathbf{5}}_{i}, \mathbf{1 0}_{i}(i=1,2,3)$, which obviously should not couple to the adjoint $\mathbf{2 4}$.

3. The two Higgs doublets of the MSSM should mostly be made out of a pair of Higgs $\overline{\mathbf{5}}+\mathbf{5}$, which couple to the third fermionic generation.

After the method of reduction of couplings is applied, the symmetry is enhanced, leading to the superpotential $[39,95]$ :

$$
\begin{aligned}
W & =\sum_{i=1}^{3}\left[\frac{1}{2} g_{i}^{u} \mathbf{1 0}_{i} \mathbf{1 0}_{i} H_{i}+g_{i}^{d} \mathbf{1 0}_{i} \overline{\mathbf{5}}_{i} \bar{H}_{i}\right]+g_{23}^{u} \mathbf{1 0}_{2} \mathbf{1 0}_{3} H_{4} \\
& +g_{23}^{d} \mathbf{1 0}_{2} \overline{\mathbf{5}}_{3} \bar{H}_{4}+g_{32}^{d} \mathbf{1 0}_{3} \overline{\mathbf{5}}_{2} \bar{H}_{4}+g_{2}^{f} H_{2} \mathbf{2 4} \bar{H}_{2}+g_{3}^{f} H_{3} \mathbf{2 4} \bar{H}_{3}+\frac{g^{\lambda}}{3}(\mathbf{2 4})^{3} .
\end{aligned}
$$

The non-degenerate and isolated solutions to $\gamma_{i}^{(1)}=0$ give:

$$
\begin{aligned}
& \left(g_{1}^{u}\right)^{2}=\frac{8}{5} g^{2},\left(g_{1}^{d}\right)^{2}=\frac{6}{5} g^{2},\left(g_{2}^{u}\right)^{2}=\left(g_{3}^{u}\right)^{2}=\frac{4}{5} g^{2}, \\
& \left(g_{2}^{d}\right)^{2}=\left(g_{3}^{d}\right)^{2}=\frac{3}{5} g^{2},\left(g_{23}^{u}\right)^{2}=\frac{4}{5} g^{2},\left(g_{23}^{d}\right)^{2}=\left(g_{32}^{d}\right)^{2}=\frac{3}{5} g^{2}, \\
& \left(g^{\lambda}\right)^{2}=\frac{15}{7} g^{2},\left(g_{2}^{f}\right)^{2}=\left(g_{3}^{f}\right)^{2}=\frac{1}{2} g^{2},\left(g_{1}^{f}\right)^{2}=0,\left(g_{4}^{f}\right)^{2}=0,
\end{aligned}
$$

and from the sum rule we obtain:

$$
m_{H_{u}}^{2}+2 m_{\mathbf{1 0}}^{2}=M^{2}, m_{H_{d}}^{2}-2 m_{\mathbf{1 0}}^{2}=-\frac{M^{2}}{3}, m_{\overline{\mathbf{5}}}^{2}+3 m_{\mathbf{1 0}}^{2}=\frac{4 M^{2}}{3} .
$$


One can observe that we have only two free parameters $\left(m_{10}\right.$ and $\left.M\right)$ for the dimensionful sector.

As it was mentioned already, after the $S U(5)$ gauge symmetry breaking we assume we have the MSSM, i.e. only two Higgs doublets. This can be done by introducing appropriate mass terms that allow for a rotation of the Higgs sector [19,23,96-98] in such a way, that only one pair of Higgs doublets, coupled mostly to the third generation, remains light and acquires vevs. To avoid fast proton decay the usual fine tuning to achieve doublet-triplet splitting is performed, although the mechanism is not identical to the one of the minimal $S U(5)$, since we now have an extended Higgs sector.

Thus, after the GUT gauge symmetry is broken, we are left with the MSSM with the boundary conditions for the third family given by the finiteness conditions. The other two families are not restricted.

\subsection{Finite $S U(N)^{3}$ Unification}

We continue examining the possibility of constructing realistic FUTs based on product gauge groups [99]. Consider an $N=1$ supersymmetric theory, with gauge group $S U(N)_{1} \times S U(N)_{2} \times$ $\cdots \times S U(N)_{k}$, with $n_{f}$ copies (number of families) of the supersymmetric multiplets $\left(N, N^{*}, 1, \ldots, 1\right)+$ $\left(1, N, N^{*}, \ldots, 1\right)+\cdots+\left(N^{*}, 1,1, \ldots, N\right)$. The one-loop $\beta$-function coefficient in the renormalizationgroup equation of each $S U(N)$ gauge coupling is simply given by

$$
b=\left(-\frac{11}{3}+\frac{2}{3}\right) N+n_{f}\left(\frac{2}{3}+\frac{1}{3}\right)\left(\frac{1}{2}\right) 2 N=-3 N+n_{f} N .
$$

This means that $n_{f}=3$ is the only solution of Eq.(5.4) that yields $b=0$. Since $b=0$ is a necessary condition for a finite field theory, the existence of three families of quarks and leptons is natural in such models, provided the matter content is exactly as given above.

The model of this type with best phenomenology is the $S U(3)^{3}$ model discussed in Ref. [99], where the details of the model are given. It corresponds to the well-known example of $S U(3)_{C} \times$ $S U(3)_{L} \times S U(3)_{R}$ [100-103], with quarks transforming as

$$
q=\left(\begin{array}{lll}
d & u & h \\
d & u & h \\
d & u & h
\end{array}\right) \sim\left(3,3^{*}, 1\right), \quad q^{c}=\left(\begin{array}{lll}
d^{c} & d^{c} & d^{c} \\
u^{c} & u^{c} & u^{c} \\
h^{c} & h^{c} & h^{c}
\end{array}\right) \sim\left(3^{*}, 1,3\right),
$$

and leptons transforming as

$$
\lambda=\left(\begin{array}{ccc}
N & E^{c} & v \\
E & N^{c} & e \\
v^{c} & e^{c} & S
\end{array}\right) \sim\left(1,3,3^{*}\right) .
$$

Switching the first and third rows of $q^{c}$ together with the first and third columns of $\lambda$, we obtain the alternative left-right model first proposed in Ref. [103] in the context of superstring-inspired $E_{6}$.

In order for all the gauge couplings to be equal at an energy scale, $M_{\mathrm{GUT}}$, the cyclic symmetry $Z_{3}$ must be imposed, i.e.

$$
q \rightarrow \lambda \rightarrow q^{c} \rightarrow q
$$

where $q$ and $q^{c}$ are given in eq. (5.5) and $\lambda$ in eq. (5.6). Then, the first of the finiteness conditions (3.9) for one-loop finiteness, namely the vanishing of the gauge $\beta$-function is satisfied. 
Next let us consider the second condition, i.e. the vanishing of the anomalous dimensions of all superfields, eq. (3.10). To do that first we have to write down the superpotential. If there is just one family, then there are only two trilinear invariants, which can be constructed respecting the symmetries of the theory, and therefore can be used in the superpotential as follows

$$
f \operatorname{Tr}\left(\lambda q^{c} q\right)+\frac{1}{6} f^{\prime} \varepsilon_{i j k} \varepsilon_{a b c}\left(\lambda_{i a} \lambda_{j b} \lambda_{k c}+q_{i a}^{c} q_{j b}^{c} q_{k c}^{c}+q_{i a} q_{j b} q_{k c}\right)
$$

where $f$ and $f^{\prime}$ are the Yukawa couplings associated to each invariant. Quark and leptons obtain masses when the scalar parts of the superfields $\left(\tilde{N}, \tilde{N}^{c}\right)$ obtain vacuum expectation values (vevs),

$$
m_{d}=f\langle\tilde{N}\rangle, m_{u}=f\left\langle\tilde{N}^{c}\right\rangle, m_{e}=f^{\prime}\langle\tilde{N}\rangle, m_{v}=f^{\prime}\left\langle\tilde{N}^{c}\right\rangle
$$

With three families, the most general superpotential contains $11 f$ couplings, and $10 f^{\prime}$ couplings, subject to 9 conditions, due to the vanishing of the anomalous dimensions of each superfield. The conditions are the following

$$
\sum_{j, k} f_{i j k}\left(f_{l j k}\right)^{*}+\frac{2}{3} \sum_{j, k} f_{i j k}^{\prime}\left(f_{l j k}^{\prime}\right)^{*}=\frac{16}{9} g^{2} \delta_{i l}
$$

where

$$
\begin{aligned}
& f_{i j k}=f_{j k i}=f_{k i j}, \\
& f_{i j k}^{\prime}=f_{j k i}^{\prime}=f_{k i j}^{\prime}=f_{i k j}^{\prime}=f_{k j i}^{\prime}=f_{j i k}^{\prime} .
\end{aligned}
$$

Quarks and leptons receive masses when the scalar part of the superfields $\tilde{N}_{1,2,3}$ and $\tilde{N}_{1,2,3}^{c}$ obtain vevs as follows

$$
\begin{array}{ll}
\left(\mathscr{M}_{d}\right)_{i j}=\sum_{k} f_{k i j}\left\langle\tilde{N}_{k}\right\rangle, & \left(\mathscr{M}_{u}\right)_{i j}=\sum_{k} f_{k i j}\left\langle\tilde{N}_{k}^{c}\right\rangle, \\
\left(\mathscr{M}_{e}\right)_{i j}=\sum_{k} f_{k i j}^{\prime}\left\langle\tilde{N}_{k}\right\rangle, & \left(\mathscr{M}_{v}\right)_{i j}=\sum_{k} f_{k i j}^{\prime}\left\langle\tilde{N}_{k}^{c}\right\rangle .
\end{array}
$$

We will assume that the below $M_{\mathrm{GUT}}$ we have the usual MSSM ${ }^{1}$, with the two Higgs doublets coupled maximally to the third generation. Therefore we have to choose the linear combinations $\tilde{N}^{c}=\sum_{i} a_{i} \tilde{N}_{i}^{c}$ and $\tilde{N}=\sum_{i} b_{i} \tilde{N}_{i}$ to play the role of the two Higgs doublets, which will be responsible for the electroweak symmetry breaking. This can be done by choosing appropriately the masses in the superpotential [98], since they are not constrained by the finiteness conditions. We choose that the two Higgs doublets are predominately coupled to the third generation. Then these two Higgs doublets couple to the three families differently, thus providing the freedom to understand their different masses and mixings. The remnants of the $S U(3)^{3}$ FUT are the boundary conditions on the gauge and Yukawa couplings, i.e. Eq.(5.10), the $h=-M C$ relation, and the soft scalar-mass sum rule eq. (4.4) at $M_{\mathrm{GUT}}$, which, when applied to the present model, takes the form

$$
m_{H_{u}}^{2}+m_{\tilde{t}^{c}}^{2}+m_{\tilde{q}}^{2}=M^{2}=m_{H_{d}}^{2}+m_{\tilde{b}^{c}}^{2}+m_{\tilde{q}}^{2}
$$

\footnotetext{
${ }^{1}$ For details of how the spontaneous breaking of $S U(3)^{3}$ to MSSM can be achieved see refs [104, 105] and refs therein.
} 
where $\tilde{t}^{c}, \tilde{b}^{c}$, and $\tilde{q}$ are the scalar parts of the corresponding superfields.

Concerning the solution to Eq.(5.10) we consider two versions of the model:

I) An all-loop finite model with a unique and isolated solution, in which $f^{\prime}$ vanishes, which leads to the following relations

$$
f^{2}=f_{111}^{2}=f_{222}^{2}=f_{333}^{2}=\frac{16}{9} g^{2} .
$$

As for the lepton masses, since all $f^{\prime}$ couplings have been fixed to be zero at this order, in principle they would be expected to appear radiatively induced by the scalar lepton masses appearing in the SSB sector of the theory. However, due to the finiteness conditions they cannot appear radiatively and remain as a problem for further study.

II) A two-loop finite solution, in which we keep $f^{\prime}$ non-vanishing and we use it to introduce the lepton masses. The model in turn becomes finite only up to two-loops since the corresponding solution of Eq.(5.10) is not an isolated one any more, i.e. it is a parametric one. In this case we have the following boundary conditions for the Yukawa couplings

$$
f^{2}=r\left(\frac{16}{9}\right) g^{2}, \quad f^{\prime 2}=(1-r)\left(\frac{8}{3}\right) g^{2},
$$

where $r$ is a free parameter which parametrizes the different solutions to the finiteness conditions. As for the boundary conditions of the soft scalars, we have the universal case.

\subsection{Reduction of Couplings in the MSSM}

In this section we are working in the framework of MSSM, assuming though the existence of a covering GUT.

The analysis of the partial reduction of couplings in this framework was first done in refs [30, 170].

The superpotential of the MSSM (where again we restrict ourselves to the third generation of fermions) is defined by

$$
W=Y_{t} H_{2} Q t^{c}+Y_{b} H_{1} Q b^{c}+Y_{\tau} H_{1} L \tau^{c}+\mu H_{1} H_{2},
$$

where $Q, L, t, b, \tau, H_{1}, H_{2}$ are the usual superfields of MSSM, while the SSB Lagrangian is given by

$$
\begin{aligned}
-\mathscr{L}_{\text {SSB }} & =\sum_{\phi} m_{\phi}^{2} \hat{\phi^{*}} \hat{\phi}+\left[m_{3}^{2} \hat{H}_{1} \hat{H}_{2}+\sum_{i=1}^{3} \frac{1}{2} M_{i} \lambda_{i} \lambda_{i}+\text { h.c }\right] \\
& +\left[h_{t} \hat{H_{2}} \hat{Q} \hat{t}^{c}+h_{b} \hat{H_{1}} \hat{Q} \hat{b}^{c}+h_{\tau} \hat{H}_{1} \hat{L} \hat{\tau}^{c}+\text { h.c. }\right],
\end{aligned}
$$

where $\hat{\phi}$ represents the scalar component of all superfields, $\lambda$ refers to the gaugino fields while all hatted fields refer to the scalar components of the corresponding superfield. The Yukawa $Y_{t, b, \tau}$ and the trilinear $h_{t, b, \tau}$ couplings refer to the third generator only, neglecting the first two generations.

Let us start with the dimensionless couplings, i.e. gauge and Yukawa. As a first step we consider only the strong coupling and the top and bottom Yukawa couplings, while the other two gauge couplings and the tau Yukawa will be treated as corrections. Following the above line, we reduce the Yukawa couplings in favour of the strong coupling $\alpha_{3}$

$$
\frac{Y_{i}^{2}}{4 \pi} \equiv \alpha_{i}=G_{i}^{2} \alpha_{3}, \quad i=t, b,
$$


and using the RGE for the Yukawa, we get

$$
G_{i}^{2}=\frac{1}{3}, \quad i=t, b .
$$

This system of the top and bottom Yukawa couplings reduced with the strong one is dictated by (i) the different running behaviour of the $S U(2)$ and $U(1)$ coupling compared to the strong one [50] and (ii) the incompatibility of applying the above reduction for the tau Yukawa since the corresponding $G^{2}$ turns negative [107]. Adding now the two other gauge couplings and the tau Yukawa in the RGE as corrections, we obtain

$$
G_{t}^{2}=\frac{1}{3}+\frac{71}{525} \rho_{1}+\frac{3}{7} \rho_{2}+\frac{1}{35} \rho_{\tau}, \quad G_{b}^{2}=\frac{1}{3}+\frac{29}{525} \rho_{1}+\frac{3}{7} \rho_{2}-\frac{6}{35} \rho_{\tau}
$$

where

$$
\rho_{1,2}=\frac{g_{1,2}^{2}}{g_{3}^{2}}=\frac{\alpha_{1,2}}{\alpha_{3}}, \quad \rho_{\tau}=\frac{g_{\tau}^{2}}{g_{3}^{2}}=\frac{\frac{Y_{\tau}^{2}}{4 \pi}}{\alpha_{3}}
$$

Note that the corrections in Eq.(5.20) are taken at the GUT scale and under the assumption that

$$
\frac{d}{d g_{3}}\left(\frac{Y_{t, b}^{2}}{g_{3}^{2}}\right)=0 .
$$

Let us comment on our assumption above, which led to the Eq.(5.20). In practice we assume that even including the corrections from the rest of the gauge as well as the tau Yukawa couplings, at the GUT scale the ratio of the top and bottom couplings $\alpha_{t, b}$ over the strong coupling are still constant, i.e. their scale dependence is negligible. Or, rephrasing it, our assumption can be understood as a requirement that in the ultraviolet (close to the GUT scale) the ratios of the top and bottom Yukawa couplings over the strong coupling become least sensitive against the change of the renormalization scale. This requirement sets the boundary condition at the GUT scale, given in Eq.(5.20). Alternatively one could follow the systematic method to include the corrections to a non-trivially reduced system developed in ref. [51], but considering two reduced systems: the first one consisting of the "top, bottom" couplings and the second of the "strong, bottom" ones.

In the next order the corrections are assumed to be in the form

$$
\alpha_{i}=G_{i}^{2} \alpha_{3}+J_{i}^{2} \alpha_{3}^{2}, \quad i=t, b
$$

Then, the coefficients $J_{i}$ are given by

$$
J_{i}^{2}=\frac{1}{4 \pi} \frac{17}{24}, \quad i=t, b
$$

for the case where only the strong gauge and the top and bottom Yukawa couplings are active, while for the case where the other two gauge and the tau Yukawa couplings are added as corrections we obtain

$$
J_{t}^{2}=\frac{1}{4 \pi} \frac{N_{t}}{D}, \quad J_{b}^{2}=\frac{1}{4 \pi} \frac{N_{b}}{5 D}
$$

where $D, N_{t}$ and $N_{b}$ are known quantities. 
We move now to the dimension-1 parameters of the SSB Lagrangian, namely the trilinear couplings $h_{t, b, \tau}$ of the SSB Lagrangian, Eq. (5.19). Again, following the pattern in the Yukawa reduction, in the first stage we reduce $h_{t, b}$, while $h_{\tau}$ will be treated as a correction.

$$
h_{i}=c_{i} Y_{i} M_{3}=c_{i} G_{i} M_{3} g_{3}, \quad i=t, b,
$$

where $M_{3}$ is the gluino mass. Using the RGE for the two $h$ we get

$$
c_{t}=c_{b}=-1,
$$

where we have also used the 1-loop relation between the gaugino mass and the gauge coupling RGE

$$
2 M_{i} \frac{d g_{i}}{d t}=g_{i} \frac{d M_{i}}{d t}, \quad i=1,2,3 .
$$

Adding the other two gauge couplings as well as the tau Yukawa $h_{\tau}$ as correction we get

$$
c_{t}=-\frac{A_{A} A_{b b}+A_{t b} B_{B}}{A_{b t} A_{t b}-A_{b b} A_{t t}}, \quad c_{b}=-\frac{A_{A} A_{b t}+A_{t t} B_{B}}{A_{b t} A_{t b}-A_{b b} A_{t t}},
$$

Again, $A_{t t}, A_{b b}$ and $A_{t b}$ are known quantities.

Finally we consider the soft squared masses $m_{\phi}^{2}$ of the SSB Lagrangian. Their reduction takes the form

$$
m_{i}^{2}=c_{i} M_{3}^{2}, \quad i=Q, u, d, H_{u}, H_{d} .
$$

For our completely reduced system (in 1-loop), i.e. $g_{3}, Y_{t}, Y_{b}, h_{t}, h_{b}$, the coefficients of the soft masses become

$$
c_{Q}=c_{u}=c_{d}=\frac{2}{3}, \quad c_{H_{u}}=c_{H_{d}}=-1 / 3,
$$

obeying the celebrated sum rules

$$
\frac{m_{Q}^{2}+m_{u}^{2}+m_{H_{u}}^{2}}{M_{3}^{2}}=c_{Q}+c_{u}+c_{H_{u}}=1, \quad \frac{m_{Q}^{2}+m_{d}^{2}+m_{H_{d}}^{2}}{M_{3}^{2}}=c_{Q}+c_{d}+c_{H_{d}}=1 .
$$

The selection of free parameters in this model, which tightly connected to the prediction of the fermion masses, will be discussed in Sect. 9.1. Subsequently, the corresponding phenomenological implications of the quark mass predictions are analyzed in 9.2.

\section{Phenomenological Constraints}

Here we outline the various constraints that are taken into account in our phenomenological analysis. We first consider four types of flavour constraints, in which SUSY is known to have significant impact ${ }^{2}$. Specifically, we consider the flavour observables $\operatorname{BR}(b \rightarrow s \gamma), \operatorname{BR}\left(B_{s} \rightarrow \mu^{+} \mu^{-}\right)$, $\operatorname{BR}\left(B_{u} \rightarrow \tau v\right)$ and $\Delta M_{B_{s}}$. It should be noted that for this review we have not used the latest experimental and theoretical values. However, this has a minor impact on the presented results. The uncertainties below are the linear combination of the experimental error and twice the theoretical uncertainty in the MSSM. The constraints are:

\footnotetext{
${ }^{2}$ Over the past years several "flavor anomalies" appeared. The most significant ones are given by the measurements of $R\left(K^{(*)}=\mathrm{BR}\left(B \rightarrow K^{(*)} \mu^{+} \mu^{-}\right) / \mathrm{BR}\left(B \rightarrow K^{(*)} e^{+} e^{-}\right)\right)$and $R\left(D^{(*)}=\mathrm{BR}\left(B \rightarrow D^{(*)} \tau v\right) / \mathrm{BR}\left(B \rightarrow D^{(*)} \mu v\right)\right)$ as well as the measurement of $P_{5}^{\prime}$ capturing the momentum dependance of the $B \rightarrow K^{*} \mu^{+} \mu^{-}$decay [108]. While (a combination of) these anomalies may turn out to be significant (see, e.g., Ref. [109]), our models do not provide any solution to them. Consequently, they do not present an additional constraint on our preferred parameter space.
} 
- For the branching ratio $\mathrm{BR}(b \rightarrow s \gamma)$ we take a value from the Heavy Flavor Averaging Group (HFAG) [110,111]:

$$
\frac{\mathrm{BR}(b \rightarrow s \gamma)^{\exp }}{\mathrm{BR}(b \rightarrow s \gamma)^{\mathrm{SM}}}=1.089 \pm 0.27
$$

- For the branching ratio $\mathrm{BR}\left(B_{s} \rightarrow \mu^{+} \mu^{-}\right)$we use a combination of CMS and LHCb data [112, 113,115-117]:

$$
\mathrm{BR}\left(B_{s} \rightarrow \mu^{+} \mu^{-}\right)=(2.9 \pm 1.4) \times 10^{-9} .
$$

- For the $B_{u}$ decay to $\tau v$ we use the limit $[111,118,119]$ :

$$
\frac{\mathrm{BR}\left(B_{u} \rightarrow \tau v\right)^{\exp }}{\mathrm{BR}\left(B_{u} \rightarrow \tau v\right)^{\mathrm{SM}}}=1.39 \pm 0.69
$$

- For $\Delta M_{B_{s}}$ we use $[120,121]$ :

$$
\frac{\Delta M_{B_{s}}^{\exp }}{\Delta M_{B_{s}}^{\mathrm{SM}}}=0.97 \pm 0.2
$$

Since the quartic couplings in the Higgs potential are given by the SM gauge couplings, the lightest Higgs boson mass is not a free parameter, but rather predicted in terms of other parameters. Higher-order corrections are crucial for a precise prediction of $M_{h}$; see Refs. [125-127] for reviews.

The discovery of a Higgs-like particle at ATLAS and CMS in July 2012 [1,2] can be interpreted as the discovery of the light $\mathscr{C} \mathscr{P}$-even Higgs boson of the MSSM Higgs spectrum [128-130]. The experimental average for the (SM) Higgs boson mass obtained at the LHC Run I is given by [131]

$$
M_{H}^{\exp }=125.1 \pm 0.3 \mathrm{GeV} .
$$

More recent Run II measurements confirm this measurement. The uncertainty, however is dominated by the theoretical accuracy for the prediction of $M_{h}$ in the MSSM, which was estimated to be at the level of $3 \mathrm{GeV}$ [132-134]. It should be noted that this estimate is only valid if the most accurate prediction of $M_{h}$ is employed. For the following phenomenological analyses the code FeynHiggs [132, 134, 135] (Version 2.14 .0 beta) was used to predict the light Higgs mass. The evaluation of the Higgs masses with FeynHiggs is based on the combination of a fixed order diagrammatic calculation and a resummation of the (sub)leading logarithmic contributions at all orders of perturbation theory. This combination ensures a reliable evaluation of $M_{h}$ also for large SUSY scales. Refinements in the combination of the fixed order log resummed calculation have been included w.r.t. previous versions [134]. They resulted in a more precise $M_{h}$ evaluation for high supersymmetric mass scales and also in a downward shift of $M_{h}$ at the level of $\mathscr{O}(2 \mathrm{GeV})$ for large SUSY masses. For our analyses we used two estimates for the theory uncertainty of 3(2) GeV. The a total uncertainty for $M_{h}$, combined of the experimental and the theoretical uncertainty, is then given by

$$
M_{h}=125.1 \pm 3.1(2.1) \mathrm{GeV} .
$$

We finally briefly comment on possible Cold Dark Matter (CDM) constraints. Since it is well known that the lightest neutralino, being the Lightest SUSY Particle (LSP), is an excellent 
candidate for CDM [122], one can in principle demand that the lightest neutralino is indeed the LSP and parameters leading to a different LSP could be discarded. The current bound, favoured by a joint analysis of WMAP/Planck and other astrophysical and cosmological data, is at $2 \sigma$ level given by $[123,124]$

$$
\Omega_{\mathrm{CDM}} h^{2}=0.1120 \pm 0.0112 .
$$

However, in the analyzed parameter space the relic abundance turns out to be too high in comparison with Eq. (6.7). Consequently, on a more general basis a mechanism is needed in our models to reduce the CDM abundance in the early universe. This issue could, for instance, be related to another problem, that of neutrino masses. Within the FUTs this type of masses cannot be generated naturally, although a non-zero value for neutrino masses has clearly been established [108]. However, the FUTs discussed here can, in principle, be easily extended by introducing bilinear R-parity violating terms that preserve finiteness and introduce the desired neutrino masses [145]. More generally, $R$-parity violation [148] would have a small impact on the collider phenomenology presented here (apart from fact the SUSY search strategies could not rely on a 'missing energy' signature), but remove the CDM bound of Eq. (6.7) completely. Consequently, Eq. (6.7) was not taken into account in the analyses presented below.

\section{Numerical Analysis of the FUT}

\subsection{FUT Predictions for Future Colliders}

Since the gauge symmetry is broken below $M_{\mathrm{GUT}}$, the finiteness conditions do not restrict the renormalization properties at low energies. Thus, all that remains are boundary conditions on the gauge and Yukawa couplings (5.2), the $h=-M C$ (4.2) relation and the soft scalar-mass sum rules at $M_{\mathrm{GUT}}$.

In Figure 1 we show the $S U(5)$-FUT predictions for $m_{t}$ and $m_{b}\left(M_{Z}\right)$ as a function of the unified gaugino mass $M$ for the cases $\mu<0$ and $\mu>0$. We use the experimental value of the top quark pole mass as in [119].

$$
m_{t}^{\exp }=(173.2 \pm 0.9) \mathrm{GeV}
$$
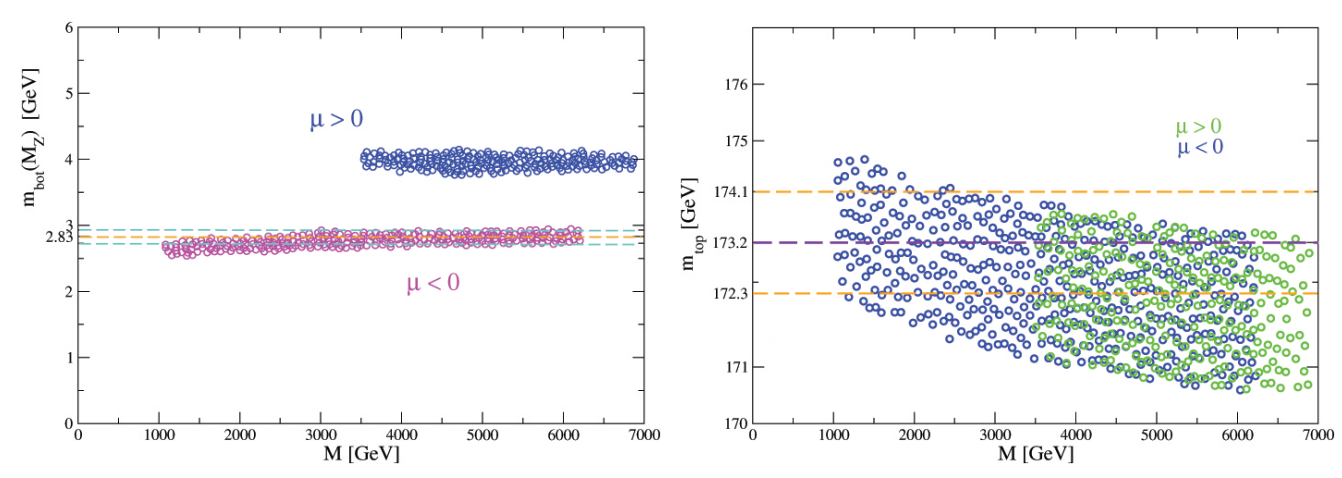

Figure 1: The bottom quark mass at the $Z$ boson scale (left) and top quark pole mass (right) as a function of $M$ for both signs of $\mu$.

We did not include the latest LHC/Tevatron data leading to $m_{t}^{\exp }=(173.34 \pm 0.76) \mathrm{GeV}[136]$, which would have a negligible impact on our analysis. 
The bottom mass is calculated at $M_{Z}$, in order to avoid uncertainties that come from running down to the pole mass. The leading supersymmetric radiative corrections to the bottom and tau masses have been taken into account [137]. For the bottom mass we use at $M_{Z}$ [119]:

$$
m_{b}\left(M_{Z}\right)=(2.83 \pm 0.10) \mathrm{GeV}
$$

The experimental bounds on the $m_{b}\left(M_{Z}\right)$ and the $m_{t}$ mass clearly single out $\mu<0$ as the only solution compatible with these constraints.

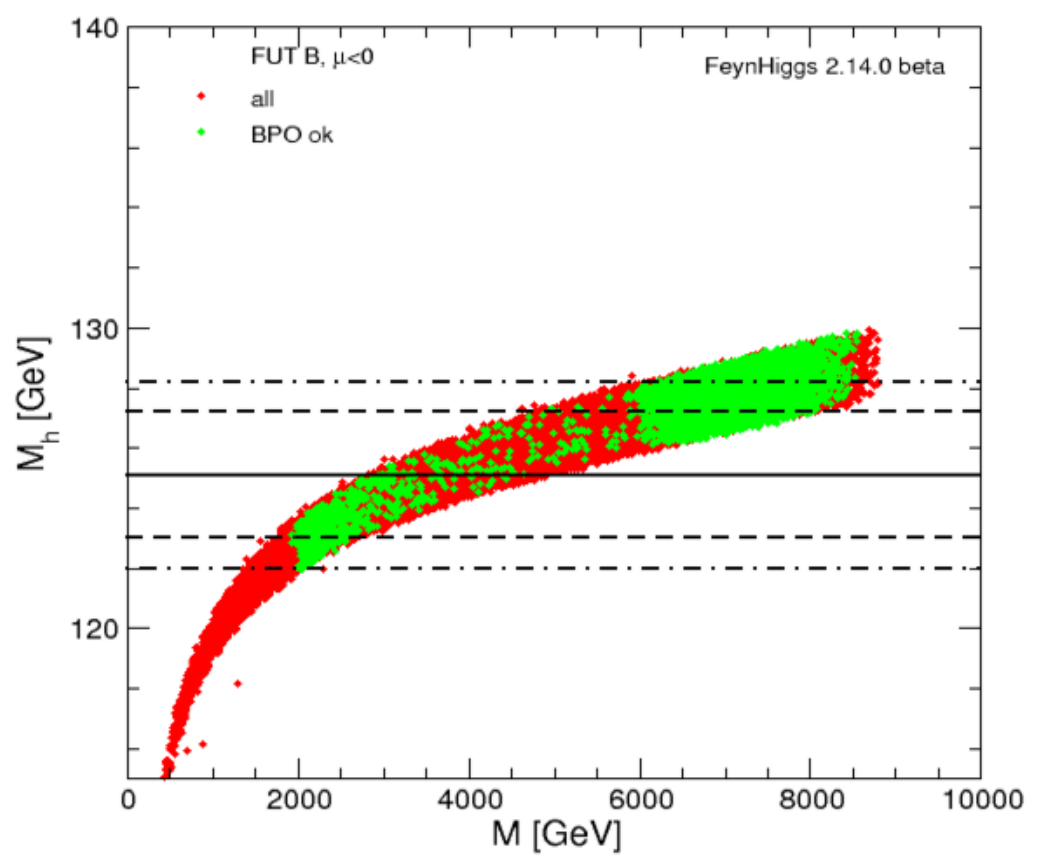

Figure 2: The lightest Higgs boson mass, $M_{h}$, as a function of $M$ for the choice $\mu<0$. The green points are the ones that satisfy the B-physics constraints.

The prediction for $M_{h}$ of $S U(5)$-FUT with $\mu<0$ is shown in Figure 2 in a range for the unified gaugino mass $0.5 \mathrm{TeV} \lesssim M \lesssim 9 \mathrm{TeV}$. The green points satisfy the $B$-physics constraints as well. We should note here that these predictions are subject to a theory uncertainty of 3 (2) GeV [132]. Older analyses, including in particular less refined evaluations of the light Higgs mass, are given in Refs. $[46,138,139]$.

The allowed values of the lightest Higgs boson mass limit the allowed supersymmetric masses' values, as it can be seen in Figure 3. In the left (right) plot we impose $M_{h}=125.1 \pm 3.1(2.1) \mathrm{GeV}$. In particular, very heavy coloured SUSY particles are favoured (nearly independent of the $M_{h}$ uncertainty), in agreement with the non-observation of those particles at the LHC [140]. Overall, the allowed coloured supersymmetric masses will remain unobservable at the (HL-)LHC, the ILC or CLIC. The lower part of the electroweak spectrum could be accessible at CLIC with $\sqrt{s}=3 \mathrm{TeV}$ The coloured spectrum would be accessible, however, at the FCC-hh [141], as could the full heavy Higgs spectrum.

In Table 1 two example spectra of $S U(5)$-FUT (with $\mu<0$ ) are shown, which span the mass range of the parameter space that is in agreement with the $B$-physics observables and the lightest 
Higgs boson mass measurement. We show the lightest and the heaviest spectrum (based on $m_{\tilde{\chi}_{1}^{0}}$ ) for $\delta M_{h}=2.1$ and $\delta M_{h}=3.1$. The Higgs boson masses are denoted as $M_{h}, M_{H}, M_{A}$ and $M_{H^{ \pm}}$. $m_{\tilde{t}_{1,2}}, m_{\tilde{t}_{1,2}}, m_{\tilde{g}}$ and $m_{\tilde{\tau}_{1,2}}$, are the scalar top, bottom, gluino and tau masses, respectively. $m_{\tilde{\chi}_{1,2}^{ \pm}}$and $m_{\tilde{\chi}_{1,2,3,4}^{0}}$ stand for chargino and neutralino masses, respectively.
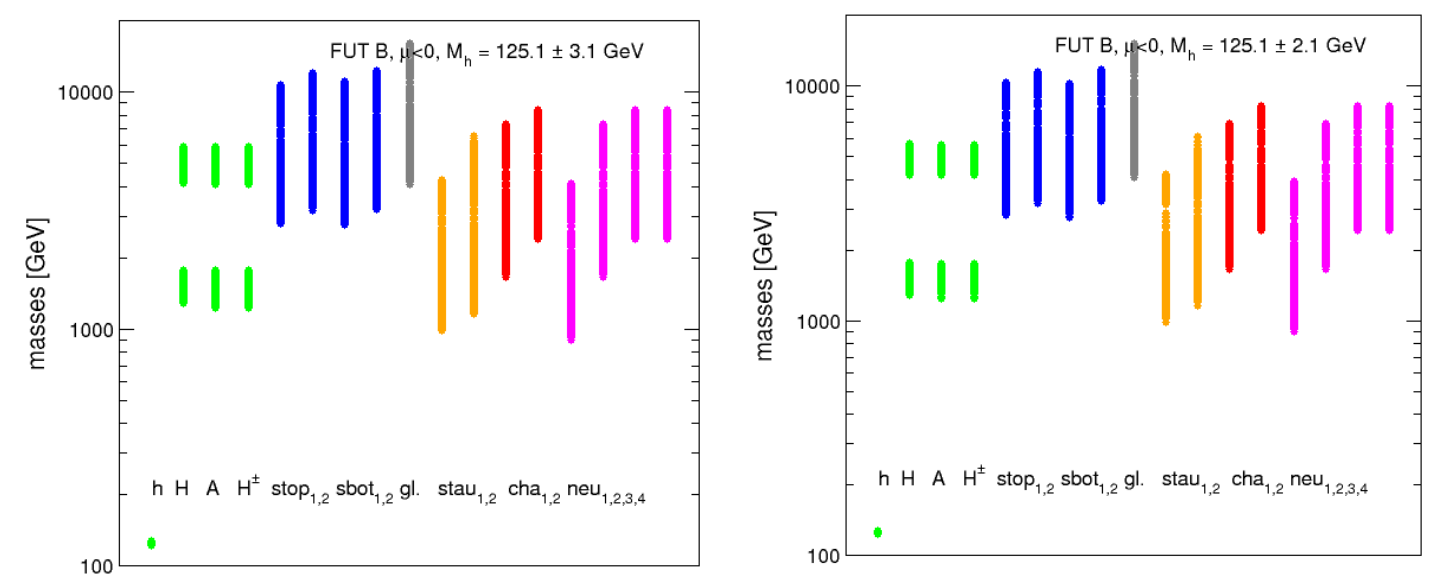

Figure 3: The (left,right) plots show the spectrum of the $S U(5)-\mathbf{F U T}$ (with $\mu<0$ ) model after imposing the constraint $M_{h}=125.1 \pm 3.1(2.1) \mathrm{GeV}$. The light (green) points are the various Higgs boson masses; the dark (blue) points following are the two scalar top and bottom masses; the gray ones are the gluino masses; then come the scalar tau masses in orange (light gray); the darker (red) points to the right are the two chargino masses; followed by the lighter shaded (pink) points indicating the neutralino masses.

No point of $S U(5)$-FUT (with $\mu<0$ ) fulfills the bound of Eq. (6.7) (we have used the code MicroMegas [142-144]). Consequently, a mechanism is needed in our model to reduce the CDM abundance in the early universe. This issue could be related to the problem of neutrino masses. These mass cannot be generated naturally within the FUT we are examining, although a nonzero value for neutrino masses has clearly been established [119]. However, SU(5)-FUT (with $\mu<0$ ) can be, in principle, extended by introducing bilinear R-parity violating terms (that preserve finiteness) and introduce neutrino masses [145-147]. R-parity violation [148] would have a small impact on the above collider phenomenology (apart from the fact that sypersymmmetry search strategies could not rely on a 'missing energy' signature), but remove the CDM bound of Eq. (6.7) completely. Other mechanisms, not involving R-parity violation and keeping the 'missing energy' signature, that could be invoked if the amount of CDM appears to be too large, concern the cosmology of the early universe. For example, "thermal inflation" [153] or "late time entropy injection" [154] can bring the CDM density into agreement with WMAP measurements.

\subsection{FUT Conclusions}

One can see that the predictions of FUT are impressive. But one could also add some comments on the theoretical side. The developments on treating the problem of divergencies include string and non-commutative theories, as well as $N=4$ supersymmetric theories [155,156], $N=8$ supergravity [157-161] and the AdS/CFT correspondence [162]. It is interesting that the $N=1$ FUT discussed here includes ideas that have survived phenomenological and theoretical tests, as well as the ultraviolet divergence problem and solves it in a minimal way. 


\begin{tabular}{|c|rrrrrrrrr|}
\hline$\delta M_{h}=2.1$ & $M_{h}$ & $M_{H}$ & $M_{A}$ & $M_{H^{ \pm}}$ & $m_{\tilde{t}_{1}}$ & $m_{\tilde{t}_{2}}$ & $m_{\tilde{b}_{1}}$ & $m_{\tilde{b}_{2}}$ & $m_{\tilde{g}}$ \\
\hline $\begin{array}{c}\text { lightest } \\
\text { heaviest }\end{array}$ & 123.1 & 1533 & 1528 & 1527 & 2800 & 3161 & 2745 & 3219 & 4077 \\
\hline & 127.2 & 4765 & 4737 & 4726 & 10328 & 11569 & 10243 & 11808 & 15268 \\
\hline $\begin{array}{c}\text { lightest } \\
\text { heaviest }\end{array}$ & 983 & 1163 & 1650 & 2414 & 900 & 1650 & 2410 & 2414 & 45 \\
\hline
\end{tabular}

\begin{tabular}{|c|rrrrrrrrr|}
\hline$\delta M_{h}=3.1$ & $M_{h}$ & $M_{H}$ & $M_{A}$ & $M_{H^{ \pm}}$ & $m_{\tilde{t}_{1}}$ & $m_{\tilde{t}_{2}}$ & $m_{\tilde{b}_{1}}$ & $m_{\tilde{b}_{2}}$ & $m_{\tilde{g}}$ \\
\hline $\begin{array}{c}\text { lightest } \\
\text { heaviest }\end{array}$ & 122.8 & 1497 & 1491 & 1490 & 2795 & 3153 & 2747 & 3211 & 4070 \\
\hline & 127.9 & 4147 & 4113 & 4103 & 10734 & 12049 & 11077 & 12296 & 16046 \\
\hline $\begin{array}{c}\text { lightest } \\
\text { heaviest }\end{array}$ & 1001 & 1172 & 1647 & 2399 & 899 & 647 & 2395 & 2399 & 44 \\
$\tilde{\tau}_{1}$ & $m_{\tilde{\chi}_{1}^{ \pm}}$ & $m_{\tilde{\chi}_{2}^{ \pm}}$ & $m_{\tilde{\chi}_{1}^{0}}$ & $m_{\tilde{\chi}_{2}^{0}}$ & $m_{\tilde{\chi}_{3}^{0}}$ & $m_{\tilde{\chi}_{4}^{0}}$ & $\tan \beta$ \\
\hline
\end{tabular}

Table 1: Two example spectra of the $S U(5)-\mathbf{F U T}($ with $\mu<0)$. All masses are in GeV and rounded to 1 (0.1) GeV (for the light Higgs mass).

In our analysis of FUT [163,164] we included restrictions of third generation quark masses and $B$-physics observables and it proved consistent with all the phenomenological constraints. Compared to our previous analyses [46,47,138,139,165,166], the improved evaluation of $M_{h}$ prefers a heavier (Higgs) spectrum and thus allows only a heavy supersymmetric spectrum. The coloured spectrum easily escapes (HL-)LHC searches, but can likely be tested at the FCC-hh. However, the lower part of the EW spectrum could be observable at CLIC.

\section{Numerical Analysis of the Finite $S U(3)^{3}$ Model}

Below $M_{\mathrm{GUT}}$ all couplings and masses of the theory run according to the RGEs of the MSSM. Thus we examine the evolution of these parameters according to their RGEs up to two-loops for dimensionless parameters and at one-loop for dimensionful ones imposing the corresponding boundary conditions. We further assume a unique SUSY breaking scale $M_{\text {SUSY }}$ and below that scale the effective theory is just the SM.

We compare our predictions with the experimental value of $m_{t}^{\exp 3}$ and recall that the theoretical values for $m_{t}$ suffer from a correction of $\sim 4 \%[14,81,167]$. In the case of the bottom quark, we take again the value evaluated at $M_{Z}$. In the case of model I, the predictions for the top quark mass

\footnotetext{
${ }^{3}$ As before, these values correspond to the experimental measurements at the time of the original evaluation. Again, the small change to the current values would not change the phenomenological analysis in a relevant way.
} 
(in this case $m_{b}$ is an input) $m_{t}$ are $\sim 183 \mathrm{GeV}$ for $\mu<0$, which is above the experimental value, and there are no solutions for $\mu>0$.
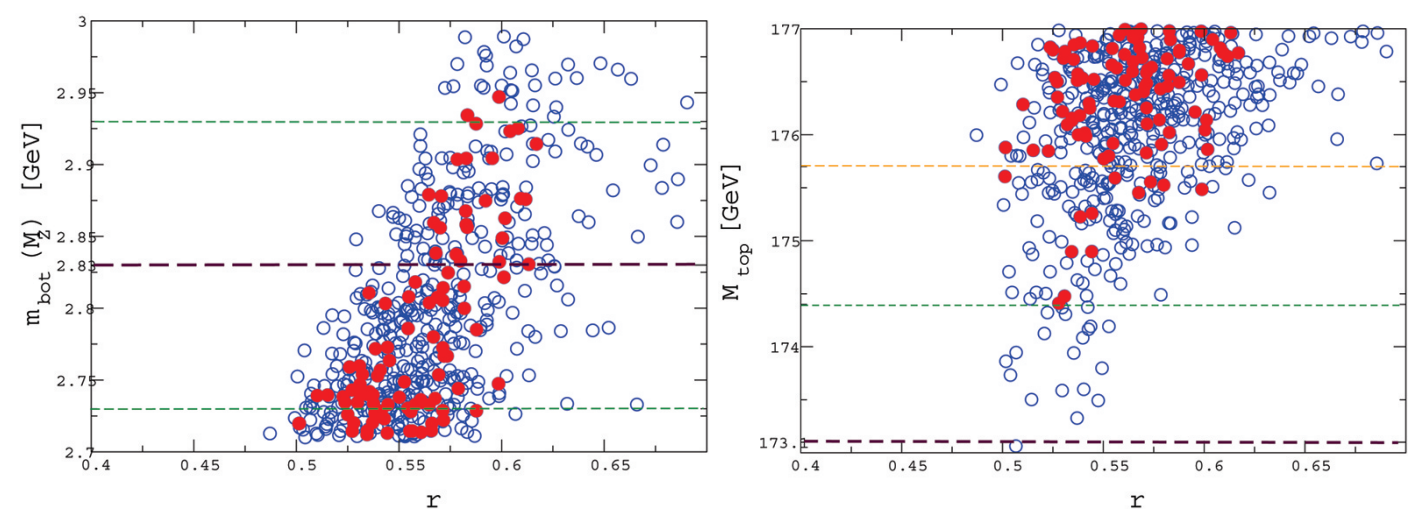

Figure 4: The figures show the values for the top and bottom quark masses for the FUT model $S U(3)^{3}$, with $\mu<0$, vs the parameter $r$. The thicker horizontal line is the experimental central value, and the lighter green and orange ones are the one and two sigma limits respectively. The red points are the ones that satisfy the $B$-physics constraints.

For the two-loop model II, we look for the values of the parameter $r$ which comply with the experimental limits given above for top and bottom quarks masses. In the case of $\mu>0$, for the bottom quark, the values of $r$ lie in the range $0.15 \lesssim r \lesssim 0.32$. For the top mass, the range of values for $\mathrm{r}$ is $0.35 \lesssim r \lesssim 0.6$. From these values we can see that there is a very small region where both top and bottom quark masses are in the experimental range for the same value of $r$. In the case of $\mu<0$ the situation is similar, although slightly better, with the range of values $0.62 \lesssim r \lesssim 0.77$ for the bottom mass, and $0.4 \lesssim r \lesssim 0.62$ for the top quark mass. In the above mentioned analysis, the masses of the new particles $h$ 's and $E$ 's of all families were taken to be at the $M_{\mathrm{GUT}}$ scale.

Taking into account new thresholds for these exotic particles below $M_{\mathrm{GUT}}$ we find a wider phenomenologically viable parameter space [168]. This can be seen in Fig. 4, where we took only one down-like exotic particle decoupling at $10^{14} \mathrm{GeV}$, below than the usual MSSM.

In this case, for $r \sim 0.5 \sim 0.62$ we have reasonable agreement with experimental data for both top and bottom quark masses, where the red points in the figure are the ones that satisfy the $B$-physics constraints (at the time of the analysis) [169]. The above analysis shows that it is worth returning with a fresh examination of this model taking into account all new experimental constraints.

\section{Numerical Analysis of the Reduced MSSM}

In this section we analyze the particle spectrum predicted by the reduced MSSM [170]. We first discuss the selection of free parameters, then apply constraints from fermion masses. Subsequently we apply the remaining constraints and discuss the observability at current and future colliders. 


\subsection{Free Parameters of the Reduced MSSM}

So far the relations among reduced parameters in terms of the fundamental ones derived in Sect. 5.3 had a part which was RGI and a another part originating from the corrections, which are scale dependent. In the analysis shown here we choose the unification scale to apply the corrections to the RGI relations. It should be noted that we are assuming a covering GUT, and thus unification of the three gauge couplings, as well as a unified gaugino mass $M$ at that scale. Also to be noted is that in the dimensionless sector of the theory, since $Y_{\tau}$ cannot be reduced in favour of the fundamental parameter $\alpha_{3}$, the mass of the $\tau$ lepton is an input parameter and consequently $\rho_{\tau}$, is an independent parameter too. At low energies, we fix the values of $\rho_{\tau}$ and $\tan \beta$ using the mass of the tau lepton $m_{\tau}\left(M_{Z}\right)=1.7462 \mathrm{GeV}$. For each value of $\rho_{\tau}$ there is a corresponding value of $\tan \beta$ that gives the appropriate $m_{\tau}\left(M_{Z}\right)$. Then we use the value found for $\tan \beta$ together with $G_{t, b}$, as obtained from the reduction equations and their respective corrections, to determine the top and bottom quark masses. We require that both the bottom and top masses are within $2 \sigma$ of their experimental value, which singles out large $\tan \beta$ values, $\tan \beta \sim 42-47$. Correspondingly, in the dimensionful sector of the theory the $\rho_{h_{\tau}}$ is a free parameter, since $h_{\tau}$ cannot be reduced in favour of the fundamental parameter $M$ (the unified gaugino mass scale). $\mu$ is a free parameter, as it cannot be reduced in favour of $M_{3}$ as discussed above. On the other hand $m_{3}^{2}$ could be reduced, but here it is chosen to leave it free. However, $\mu$ and $m_{3}^{2}$ are restricted from the requirement of EWSB, and only $\mu$ is taken as an independent parameter. Finally, the other parameter in the Higgs-boson sector, the $\mathscr{C} \mathscr{P}$-odd Higgs-boson mass $M_{A}$ is evaluated from $\mu$, as well as from $m_{H_{u}}^{2}$ and $m_{H_{d}}^{2}$, which are obtained from the reduction equations. In total we vary the parameters $\rho_{\tau}, \rho_{h_{\tau}}, M$ and $\mu$.

\subsection{Constraints from Fermion Masses}

The first step of the numerical analysis concerns the top and the bottom quark masses. As mentioned above, the variation of $\rho_{\tau}$ yields the values of $m_{t}$ (the top pole mass) and $m_{b}\left(M_{Z}\right)$, the running bottom quark mass at the $Z$ boson mass scale, where scan points which are not within $2 \sigma$ of the experimental data are neglected. This is shown in Fig. 5 [170]. The experimental values are indicated by the horizontal lines and are taken to be [119]

$$
m_{t}=173.34 \pm 1.52 \mathrm{GeV}, \quad m_{b}\left(M_{Z}\right)=2.83 \pm 0.1 \mathrm{GeV},
$$

with the uncertainties at the $2 \sigma$ level. One can see that the scan yields many parameter points that are in very good agreement with the experimental data. At the same time also the flavor constraints, see Sect. 6 are applied and shown as green dots. One can see that they are in good agreement with the measurements of the quark masses and give restrictions in the allowed ranges of $M$ (the common gaugino mass at the unification scale).

\subsection{Predictions of the reduced MSSM for future colliders}

As the next step the lightest MSSM Higgs-boson mass is evaluated. The prediction for $M_{h}$ is shown in Fig. 6 [170] as a function of $M$ in the range $1 \mathrm{TeV} \lesssim M \lesssim 6 \mathrm{TeV}$. The lightest Higgs mass ranges in

$$
M_{h} \sim 124-129 \mathrm{GeV}
$$



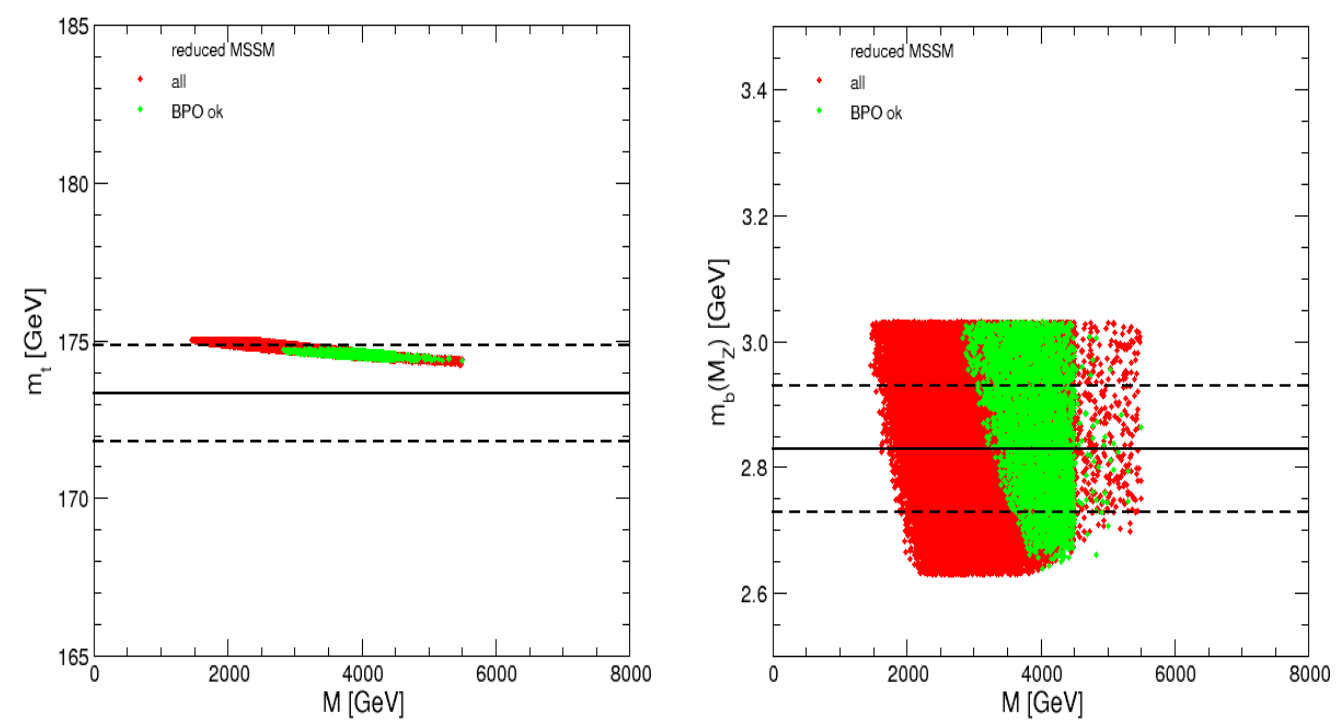

Figure 5: The left (right) plot shows our results within the reduced MSSM for the top (bottom) quark mass. The horizontal lines indicate the experimental values as given in Eq. (9.1). Taken from Ref. [170].

where we discard the "spreaded" points with possibly lower masses, which result from a numerical instability in the Higgs-boson mass calculation. One should keep in mind that these predictions are subject to a theory uncertainty of $3(2) \mathrm{GeV}$, see Sect. 6. The red points correspond to the full parameter scan, whereas the green points are the subset that is in agreement with the $B$-physics observables as discussed above (which do not exhibit any numerical instability). The inclusion of the flavor observables shifts the lower bound for $M_{h}$ up to $\sim 126 \mathrm{GeV}$.

The horizontal lines in Fig. 6 show the central value of the experimental measurement (solid), the $\pm 2.1 \mathrm{GeV}$ uncertainty (dashed) and the $\pm 3.1 \mathrm{GeV}$ uncertainty (dot-dashed). The requirement to obtain a light Higgs boson mass value in the correct range yields an upper limit on $M$ of about 5 (4) $\mathrm{TeV}$ for $M_{h}=125.1 \pm 2.1(3.1) \mathrm{GeV}$.

Naturally the $M_{h}$ limit also sets an upper limit on the low-energy SUSY masses. This turns the reduced MSSM into a highly predictive and testable theory. The full particle spectrum of the reduced MSSM (where we restricted ourselves as before to the third generation of sfermions) compliant with the $B$-physics observables is shown in Fig. 7 [170]. In the left (right) plot we impose $M_{h}=125.1 \pm 3.1(2.1) \mathrm{GeV}$. Including the Higgs mass constraints in general favours the somewhat higher part of the SUSY particle mass spectra. The tighter $M_{h}$ range cuts off the very high SUSY mass scales.

The Higgs spectrum will be fully testable at the HL-LHC, which for $\tan \beta \gtrsim 40$ can explore masses up to $\sim 2 \mathrm{TeV}$ via the channel $p p \rightarrow H / A \rightarrow \tau^{+} \tau^{-}$[171]. However, such observations would be in agreement also with a pure 2HDM, and additional observation of the SUSY particles will be necessary to analyze the model.

The lighter SUSY particles are given by the electroweak spectrum, which starts around $\sim$ 1.3 TeV. They will mostly remain unobservable at the LHC and at future $e^{+} e^{-}$colliders such as 


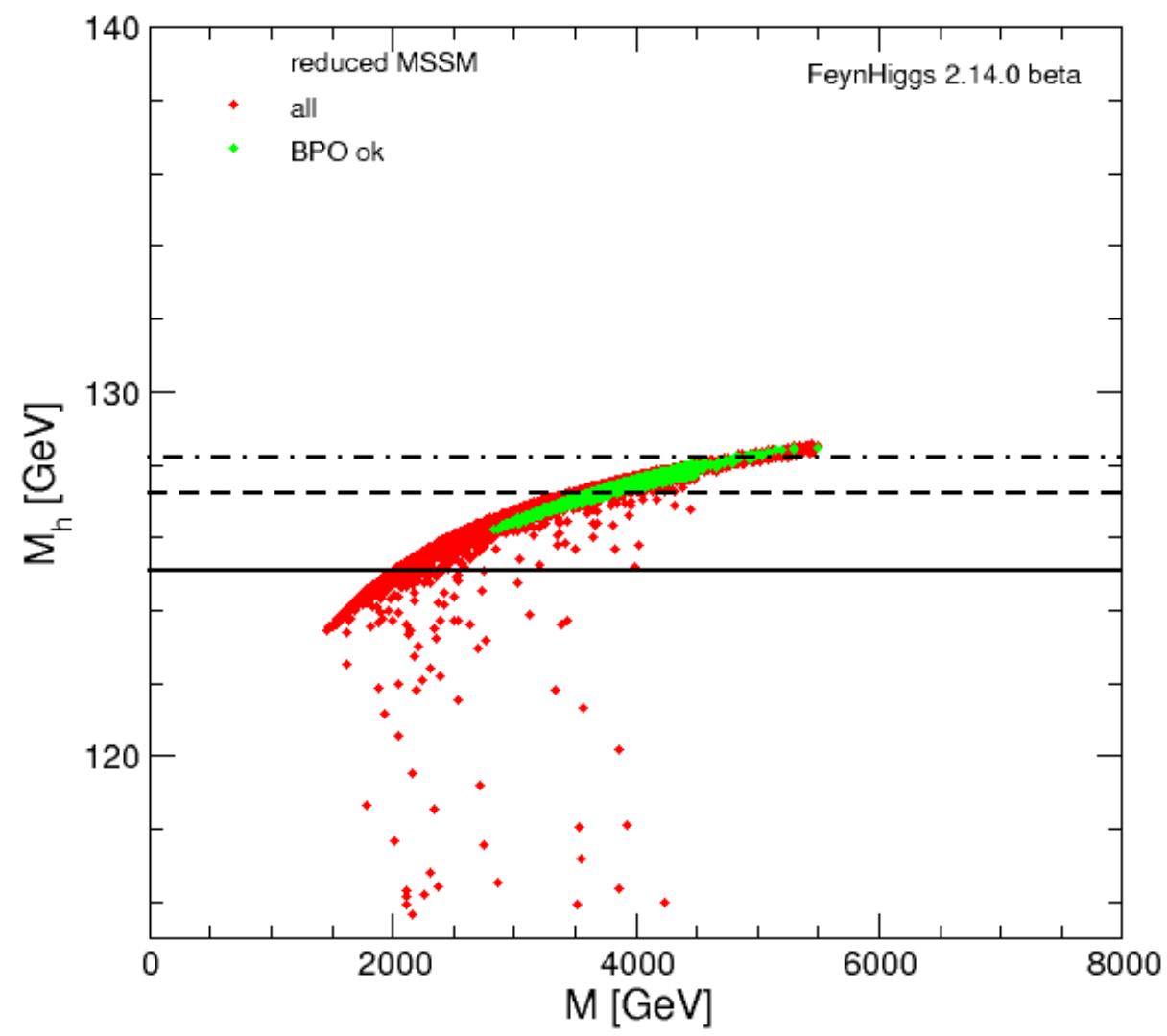

Figure 6: The lightest Higgs boson mass, $M_{h}$, as a function of $M$ (the common gaugino mass at the unification scale) in the reduced MSSM. The red points is the full model prediction. The green points fulfill the B-physics constraints (see text). Taken from Ref. [170].

the ILC or CLIC, with only the very lower range mass range below $\sim 1.5 \mathrm{TeV}$ might be observable at CLIC (with $\sqrt{s}=3 \mathrm{TeV}$ ). The coloured mass spectrum starts at around $\sim 4 \mathrm{TeV}$, which will remain unobservable at the (HL-)LHC. However, the coloured spectrum would be accessible at the FCC-hh [141]. This collider could definitely confirm the SUSY spectrum of the reduced MSSM or rule out this model.

In Tab. 2 we show three example spectra of the reduced MSSM, which span the mass range of the parameter space that is in agreement with the $B$-physics observables and the Higgs-boson mass measurement (using the same notation as in Tab. 1). The rows labelled "light" correspond to the spectrum with the smallest $m_{\tilde{\chi}_{1}^{0}}$ value (which is independent of upper limit in $M_{h}$ ). This point is an example for the lowest $M_{h}$ values that we can reach in our scan. As discussed above, the heavy Higgs boson spectrum starts above $1.4 \mathrm{TeV}$, which can be covered at the HL-LHC. The coloured spectrum is found between $\sim 4 \mathrm{TeV}$ and $\sim 6 \mathrm{TeV}$, outside the range of the (HLLHC. The LSP has a mass of $m_{\tilde{\chi}_{1}^{0}}=1339$, which might offer the possibility of $e^{+} e^{-} \rightarrow \tilde{\chi}_{1}^{0} \tilde{\chi}_{1}^{0} \gamma$ at CLIC. All other electroweak particles are too heavy to be produced at CLIC or the (HL-)LHC. 

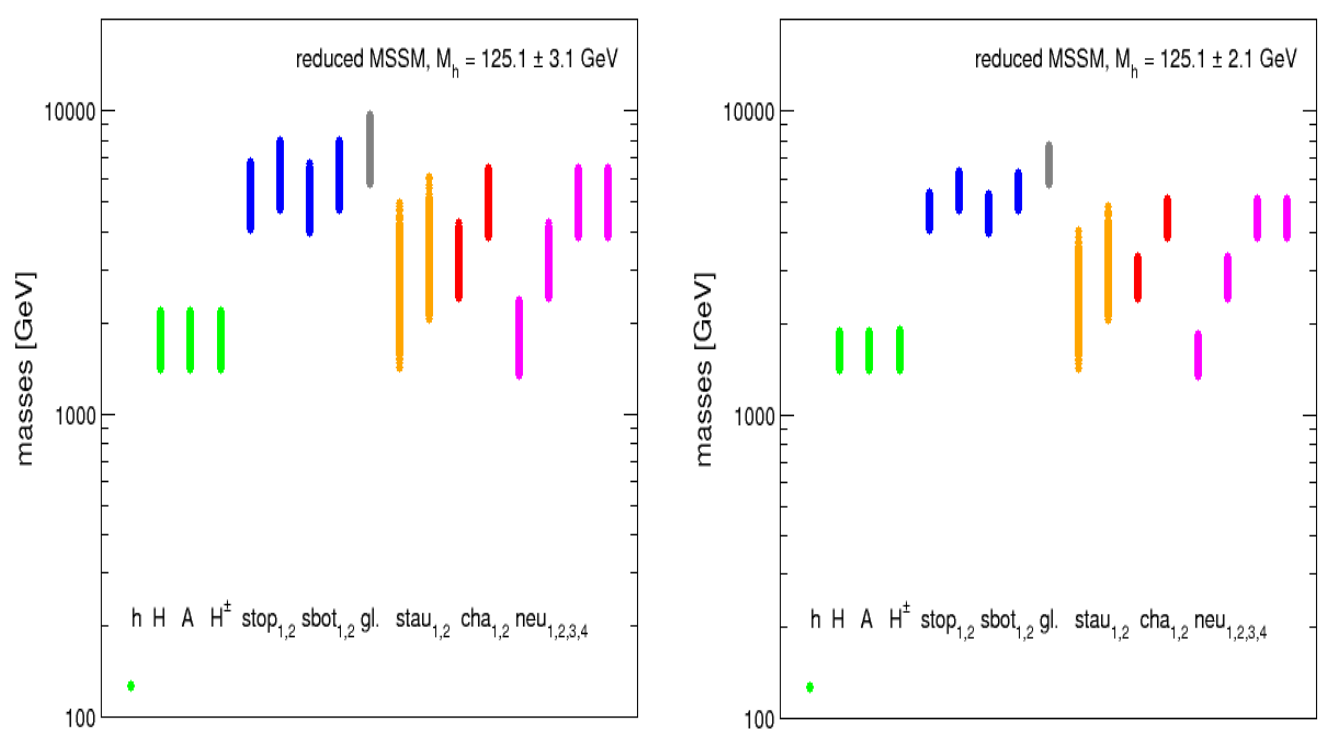

Figure 7: The left (right) plot shows the spectrum of the reduced MSSM after imposing the constraint $M_{h}=125.1 \pm 3.1(2.1) \mathrm{GeV}$. The points shown are in agreement with the B-physics observables. The light (green) points on the left are the various Higgs boson masses. The dark (blue) points following are the two scalar top and bottom masses, followed by the lighter (gray) gluino mass. Next come the lighter (beige) scalar tau masses. The darker (red) points to the right are the two chargino masses followed by the lighter shaded (pink) points indicating the neutralino masses.

\begin{tabular}{|c|rrrrrrrrr|}
\hline & $M_{h}$ & $M_{H}$ & $M_{A}$ & $M_{H^{ \pm}}$ & $m_{\tilde{t}_{1}}$ & $m_{\tilde{t}_{2}}$ & $m_{\tilde{b}_{1}}$ & $m_{\tilde{b}_{2}}$ & $m_{\tilde{s}}$ \\
\hline light & 126.2 & 1433 & 1433 & 1446 & 4052 & 4736 & 3989 & 4723 & 5789 \\
$\delta M_{h}=2.1$ & 127.2 & 1570 & 1570 & 1572 & 5361 & 6289 & 5282 & 6279 & 7699 \\
$\delta M_{h}=3.1$ & 128.1 & 1886 & 1886 & 1888 & 6762 & 7951 & 6653 & 7943 & 9683 \\
\hline
\end{tabular}

\begin{tabular}{|c|rrrrrrrrr|}
\hline & $m_{\tilde{\tau}_{1}}$ & $m_{\tilde{\tau}_{2}}$ & $m_{\tilde{\chi}_{1}^{ \pm}}$ & $m_{\tilde{\chi}_{2}^{ \pm}}$ & $m_{\tilde{\chi}_{1}^{0}}$ & $m_{\tilde{\chi}_{2}^{0}}$ & $m_{\tilde{\chi}_{3}^{0}}$ & $m_{\tilde{\chi}_{4}^{0}}$ & $\tan b$ \\
\hline light & 1906 & 2066 & 2430 & 3867 & 1339 & 2430 & 3864 & 3866 & 42.6 \\
$\delta M_{h}=2.1$ & 1937 & 2531 & 3299 & 5166 & 1833 & 3299 & 5114 & 5116 & 43.1 \\
$\delta M_{h}=3.1$ & 3153 & 3490 & 4248 & 6464 & 2376 & 4248 & 6462 & 6464 & 45.2 \\
\hline
\end{tabular}

Table 2: Three example spectra of the reduced MSSM. "light" has the smallest $m_{\tilde{\chi}_{1}^{0}}$ in our sample, " $\delta M_{h}=$ 2.1(3.1)" has the largest $m_{\tilde{\chi}_{1}^{0}}$ for $M_{h} \leq 125.1+2.1(3.1) \mathrm{GeV}$. All masses are in GeV and rounded to 1 (0.1) GeV (for the light Higgs mass).

" $\delta M_{h}=2.1(3.1)$ " has the largest $m_{\tilde{\chi}_{1}^{0}}$ for $M_{h} \leq 125.1+2.1(3.1) \mathrm{GeV}$. While, following the mass relations in the reduced MSSM, the mass spectra are substantially heavier than in the "light" case, 
one can also observe that the smaller upper limit on $M_{h}$ results in substantially lower upper limits on the various SUSY and Higgs-boson masses. In both cases the heavy Higgs spectrum is within the reach of the HL-LHC, as mentioned above. However, even in the case of $\delta M_{h}=2.1 \mathrm{GeV}$, all SUSY particles are outside the reach of the (HL-)LHC and CLIC. On the other hand, all spectra offer good possibilities for their discovery at the FCC-hh [141], as discussed above.

\subsection{Reduced MSSM Conclusions}

The reduced MSSM naturally results in a light Higgs boson in the mass range measured at the LHC. Only the Higgs sector can be tested at the HL-LHC. On the other hand, the rest of the SUSY spectrum will remain (likely) unaccessible at the (HL-)LHC, ILC and CLIC, where such a heavy spectrum also results in SM-like light Higgs boson, in agreement with LHC measurements [172]. In other words, the model is naturally in full agreement with all LHC measurements. It can be tested definitely at the FCC-hh, where large parts of the SUSY spectrum would be in the kinematic reach.

\section{References}

[1] G. Aad et al. [ATLAS Collaboration], Phys. Lett. B 716 (2012) 1 doi:10.1016/j.physletb.2012.08.020 [arXiv:1207.7214 [hep-ex]].

[2] S. Chatrchyan et al. [CMS Collaboration], Phys. Lett. B 716 (2012) 30 doi:10.1016/j.physletb.2012.08.021 [arXiv:1207.7235 [hep-ex]].

[3] G. Aad et al. [ATLAS and CMS Collaborations], JHEP 1608 (2016) 045 doi:10.1007/JHEP08(2016)045 [arXiv:1606.02266 [hep-ex]].

[4] J. C. Pati and A. Salam, Phys. Rev. Lett. 31 (1973) 661. doi:10.1103/PhysRevLett.31.661

[5] H. Georgi and S. L. Glashow, Phys. Rev. Lett. 32 (1974) 438. doi:10.1103/PhysRevLett.32.438

[6] H. Georgi, H. R. Quinn and S. Weinberg, Phys. Rev. Lett. 33 (1974) 451. doi:10.1103/PhysRevLett.33.451

[7] H. Georgi, C. E. Carlson, New York 1975, 688p

[8] H. Fritzsch and P. Minkowski, Annals Phys. 93 (1975) 193. doi:10.1016/0003-4916(75)90211-0

[9] S. Dimopoulos and H. Georgi, Nucl. Phys. B 193 (1981) 150. doi:10.1016/0550-3213(81)90522-8

[10] N. Sakai, Z. Phys. C 11 (1981) 153. doi:10.1007/BF01573998

[11] U. Amaldi, W. de Boer and H. Furstenau, Phys. Lett. B 260 (1991) 447. doi:10.1016/0370-2693(91)91641-8

[12] A. J. Buras, J. R. Ellis, M. K. Gaillard and D. V. Nanopoulos, Nucl. Phys. B 135 (1978) 66. doi:10.1016/0550-3213(78)90214-6

[13] J. Kubo, M. Mondragon, M. Olechowski and G. Zoupanos, Nucl. Phys. B 479 (1996) 25 doi:10.1016/0550-3213(96)00433-6 [hep-ph/9512435].

[14] J. Kubo, M. Mondragon and G. Zoupanos, Acta Phys. Polon. B 27 (1997) 3911 [hep-ph/9703289].

[15] T. Kobayashi, J. Kubo, M. Mondragon and G. Zoupanos, Acta Phys. Polon. B 30 (1999) 2013.

[16] P. Fayet, Nucl. Phys. B 149 (1979) 137. doi:10.1016/0550-3213(79)90162-7 
[17] W. Zimmermann, Commun. Math. Phys. 97 (1985) 211. doi:10.1007/BF01206187

[18] R. Oehme and W. Zimmermann, Commun. Math. Phys. 97 (1985) 569. doi:10.1007/BF01221218

[19] D. Kapetanakis, M. Mondragon and G. Zoupanos, Z. Phys. C 60 (1993) 181 doi:10.1007/BF01650445 [hep-ph/9210218].

[20] J. Kubo, M. Mondragon and G. Zoupanos, Nucl. Phys. B 424 (1994) 291. doi:10.1016/0550-3213(94)90296-8

[21] J. Kubo, M. Mondragon, N. D. Tracas and G. Zoupanos, Phys. Lett. B 342 (1995) 155 doi:10.1016/0370-2693(94)01396-T [hep-th/9409003].

[22] J. Kubo, M. Mondragon, M. Olechowski and G. Zoupanos, hep-ph/9510279.

[23] M. Mondragon and G. Zoupanos, Nucl. Phys. Proc. Suppl. 37C (1995) no.3, 98. doi:10.1016/0920-5632(94)00793-U

[24] J. Kubo, M. Mondragon and G. Zoupanos, Phys. Lett. B 389 (1996) 523 doi:10.1016/S0370-2693(96)01323-8 [hep-ph/9609218].

[25] O. Piguet and K. Sibold, Int. J. Mod. Phys. A 1 (1986) 913. doi:10.1142/S0217751X86000332

[26] C. Lucchesi, O. Piguet and K. Sibold, Helv. Phys. Acta 61 (1988) 321.

[27] C. Lucchesi and G. Zoupanos, Fortsch. Phys. 45 (1997) 129 [hep-ph/9604216].

[28] I. Jack and D. R. T. Jones, Phys. Lett. B 349 (1995) 294 doi:10.1016/0370-2693(95)00271-L [hep-ph/9501395].

[29] W. Zimmermann, Commun. Math. Phys. 219 (2001) 221. doi:10.1007/s002200100396

[30] M. Mondragon, N. D. Tracas and G. Zoupanos, Phys. Lett. B 728 (2014) 51 doi:10.1016/j.physletb.2013.11.043 [arXiv:1309.0996 [hep-ph]].

[31] S. Heinemeyer, J. Kubo, M. Mondragon, O. Piguet, K. Sibold, W. Zimmermann and G. Zoupanos, Honorary Volume for Wolfhart Zimmermann, Ed. Klaus Sibold. Published in PoS(Higgs \& top)001. A short version is published in arXiv:1411.7155 [hep-ph].

[32] D. R. T. Jones, L. Mezincescu and Y. P. Yao, Phys. Lett. 148B (1984) 317. doi:10.1016/0370-2693(84)90095-9

[33] I. Jack and D. R. T. Jones, Phys. Lett. B 333 (1994) 372 doi:10.1016/0370-2693(94)90156-2 [hep-ph/9405233].

[34] L. E. Ibanez and D. Lust, Nucl. Phys. B 382 (1992) 305 doi:10.1016/0550-3213(92)90189-I [hep-th/9202046].

[35] V. S. Kaplunovsky and J. Louis, Phys. Lett. B 306 (1993) 269 doi:10.1016/0370-2693(93)90078-V [hep-th/9303040].

[36] A. Brignole, L. E. Ibanez and C. Munoz, Nucl. Phys. B 422 (1994) 125 Erratum: [Nucl. Phys. B 436 (1995) 747] doi:10.1016/0550-3213(94)00600-J, 10.1016/0550-3213(94)00068-9 [hep-ph/9308271].

[37] J. A. Casas, A. Lleyda and C. Munoz, Phys. Lett. B 380 (1996) 59 doi:10.1016/0370-2693(96)00489-3 [hep-ph/9601357].

[38] Y. Kawamura, T. Kobayashi and J. Kubo, Phys. Lett. B 405 (1997) 64 doi:10.1016/S0370-2693(97)00618-7 [hep-ph/9703320]. 
[39] T. Kobayashi, J. Kubo, M. Mondragon and G. Zoupanos, Nucl. Phys. B 511 (1998) 45 doi:10.1016/S0550-3213(97)00765-7 [hep-ph/9707425].

[40] T. Kobayashi, J. Kubo and G. Zoupanos, Phys. Lett. B 427 (1998) 291 doi:10.1016/S0370-2693(98)00343-8 [hep-ph/9802267].

[41] V. A. Novikov, M. A. Shifman, A. I. Vainshtein and V. I. Zakharov, Nucl. Phys. B 229 (1983) 407. doi:10.1016/0550-3213(83)90340-1

[42] V. A. Novikov, M. A. Shifman, A. I. Vainshtein and V. I. Zakharov, Phys. Lett. 166B (1986) 329 [Sov. J. Nucl. Phys. 43 (1986) 294] [Yad. Fiz. 43 (1986) 459]. doi:10.1016/0370-2693(86)90810-5

[43] M. A. Shifman, Int. J. Mod. Phys. A 11 (1996) 5761 doi:10.1142/S0217751X96002650 [hep-ph/9606281].

[44] J. Kubo, M. Mondragon, S. Shoda and G. Zoupanos, Nucl. Phys. B 469 (1996) 3 doi:10.1016/0550-3213(96)00148-4 [hep-ph/9512258].

[45] S. Heinemeyer, M. Mondragon and G. Zoupanos, JHEP 0807 (2008) 135 doi:10.1088/1126-6708/2008/07/135 [arXiv:0712.3630 [hep-ph]].

[46] S. Heinemeyer, M. Mondragon and G. Zoupanos, Phys. Lett. B 718 (2013) 1430 doi:10.1016/j.physletb.2012.12.042 [arXiv:1211.3765 [hep-ph]].

[47] S. Heinemeyer, M. Mondragon and G. Zoupanos, Fortsch. Phys. 61 (2013) no.11, 969 doi:10.1002/prop.201300017 [arXiv:1305.5073 [hep-ph]].

[48] S. Heinemeyer, M. Mondragon and G. Zoupanos, Int. J. Mod. Phys. A 29 (2014) 1430032 doi:10.1142/S0217751X14300324 [arXiv:1412.5766 [hep-ph]].

[49] R. Oehme, Prog. Theor. Phys. Suppl. 86 (1986) 215. doi:10.1143/PTPS.86.215

[50] J. Kubo, K. Sibold and W. Zimmermann, Nucl. Phys. B 259 (1985) 331. doi:10.1016/0550-3213(85)90639-X

[51] J. Kubo, K. Sibold and W. Zimmermann, Phys. Lett. B 220 (1989) 185. doi:10.1016/0370-2693(89)90034-8

[52] O. Piguet and K. Sibold, Phys. Lett. B 229 (1989) 83. doi:10.1016/0370-2693(89)90160-3

[53] P. Breitenlohner and D. Maison, Commun. Math. Phys. 219 (2001) 179. doi:10.1007/s002200100395

[54] J. Wess and B. Zumino, Phys. Lett. 49B (1974) 52. doi:10.1016/0370-2693(74)90578-4

[55] J. Iliopoulos and B. Zumino, Nucl. Phys. B 76 (1974) 310. doi:10.1016/0550-3213(74)90388-5

[56] K. Fujikawa and W. Lang, Nucl. Phys. B 88 (1975) 61. doi:10.1016/0550-3213(75)90525-8

[57] A. Parkes and P. C. West, Phys. Lett. 138B (1984) 99. doi:10.1016/0370-2693(84)91881-1

[58] S. Rajpoot and J. G. Taylor, Phys. Lett. 147B (1984) 91. doi:10.1016/0370-2693(84)90598-7

[59] S. Rajpoot and J. G. Taylor, Int. J. Theor. Phys. 25 (1986) 117. doi:10.1007/BF00677701

[60] P. C. West, Phys. Lett. 137B (1984) 371. doi:10.1016/0370-2693(84)91734-9

[61] D. R. T. Jones and L. Mezincescu, Phys. Lett. 138B (1984) 293. doi:10.1016/0370-2693(84)91663-0

[62] D. R. T. Jones and A. J. Parkes, Phys. Lett. 160B (1985) 267. doi:10.1016/0370-2693(85)91324-3

[63] A. J. Parkes, Phys. Lett. 156B (1985) 73. doi:10.1016/0370-2693(85)91357-7 
[64] L. O’Raifeartaigh, Nucl. Phys. B 96 (1975) 331. doi:10.1016/0550-3213(75)90585-4

[65] P. Fayet and J. Iliopoulos, Phys. Lett. 51B (1974) 461. doi:10.1016/0370-2693(74)90310-4

[66] C. Lucchesi, O. Piguet and K. Sibold, Phys. Lett. B 201 (1988) 241. doi:10.1016/0370-2693(88)90221-3

[67] S. Ferrara and B. Zumino, Nucl. Phys. B 87 (1975) 207. doi:10.1016/0550-3213(75)90063-2

[68] O. Piguet and K. Sibold, Nucl. Phys. B 196 (1982) 428. doi:10.1016/0550-3213(82)90499-0

[69] O. Piguet and K. Sibold, Nucl. Phys. B 196 (1982) 447. doi:10.1016/0550-3213(82)90500-4

[70] O. Piguet and K. Sibold, Phys. Lett. B 177 (1986) 373. doi:10.1016/0370-2693(86)90770-7

[71] P. Ensign and K. T. Mahanthappa, Phys. Rev. D 36 (1987) 3148. doi:10.1103/PhysRevD.36.3148

[72] O. Piguet, hep-th/9606045.

[73] R. Oehme, K. Sibold and W. Zimmermann, Phys. Lett. 147B (1984) 115.

[74] T. P. Cheng, E. Eichten and L. F. Li, Phys. Rev. D 9 (1974) 2259.

[75] N. P. Chang, Phys. Rev. D 10 (1974) 2706.

[76] W. Zimmermann, Phys. Lett. B 311 (1993) 249.

[77] L. Alvarez-Gaume and P. H. Ginsparg, Nucl. Phys. B 243 (1984) 449. doi:10.1016/0550-3213(84)90487-5

[78] W. A. Bardeen and B. Zumino, Nucl. Phys. B 244 (1984) 421. doi:10.1016/0550-3213(84)90322-5

[79] B. Zumino, Y. S. Wu and A. Zee, Nucl. Phys. B 239 (1984) 477. doi:10.1016/0550-3213(84)90259-1

[80] R. G. Leigh and M. J. Strassler, Nucl. Phys. B 447 (1995) 95 doi:10.1016/0550-3213(95)00261-P [hep-th/9503121].

[81] M. Mondragon and G. Zoupanos, Acta Phys. Polon. B 34 (2003) 5459.

[82] R. Delbourgo, Nuovo Cim. A 25 (1975) 646. doi:10.1007/BF02729305

[83] A. Salam and J. A. Strathdee, Nucl. Phys. B 86 (1975) 142. doi:10.1016/0550-3213(75)90078-4

[84] M. T. Grisaru, W. Siegel and M. Rocek, Nucl. Phys. B 159 (1979) 429. doi:10.1016/0550-3213(79)90344-4

[85] L. Girardello and M. T. Grisaru, Nucl. Phys. B 194 (1982) 65. doi:10.1016/0550-3213(82)90512-0

[86] J. Hisano and M. A. Shifman, Phys. Rev. D 56 (1997) 5475 doi:10.1103/PhysRevD.56.5475 [hep-ph/9705417].

[87] I. Jack and D. R. T. Jones, Phys. Lett. B 415 (1997) 383 doi:10.1016/S0370-2693(97)01277-X [hep-ph/9709364].

[88] L. V. Avdeev, D. I. Kazakov and I. N. Kondrashuk, Nucl. Phys. B 510 (1998) 289 doi:10.1016/S0550-3213(98)81015-8, 10.1016/S0550-3213(97)00706-2 [hep-ph/9709397].

[89] D. I. Kazakov, Phys. Lett. B 449 (1999) 201 doi:10.1016/S0370-2693(99)00086-6 [hep-ph/9812513].

[90] D. I. Kazakov, Phys. Lett. B 421 (1998) 211 doi:10.1016/S0370-2693(97)01561-X [hep-ph/9709465]. 
[91] I. Jack, D. R. T. Jones and A. Pickering, Phys. Lett. B 426 (1998) 73 doi:10.1016/S0370-2693(98)00270-6 [hep-ph/9712542].

[92] I. Jack and D. R. T. Jones, Phys. Lett. B 465 (1999) 148 doi:10.1016/S0370-2693(99)01064-3 [hep-ph/9907255].

[93] T. Kobayashi, J. Kubo, M. Mondragon and G. Zoupanos, AIP Conf. Proc. 490 (1999) no.1, 279. doi:10.1063/1.1301389

[94] A. Karch, T. Kobayashi, J. Kubo and G. Zoupanos, Phys. Lett. B 441 (1998) 235 doi:10.1016/S0370-2693(98)01182-4 [hep-th/9808178].

[95] M. Mondragon and G. Zoupanos, J. Phys. Conf. Ser. 171 (2009) 012095. doi:10.1088/1742-6596/171/1/012095

[96] S. Hamidi and J. H. Schwarz, Phys. Lett. 147B (1984) 301. doi:10.1016/0370-2693(84)90121-7

[97] D. R. T. Jones and S. Raby, Phys. Lett. 143B (1984) 137. doi:10.1016/0370-2693(84)90820-7

[98] J. Leon, J. Perez-Mercader, M. Quiros and J. Ramirez-Mittelbrunn, Phys. Lett. 156B (1985) 66. doi:10.1016/0370-2693(85)91356-5

[99] E. Ma, M. Mondragón, and G. Zoupanos, JHEP 12, 026 (2004), hep-ph/0407236.

[100] A. De Rújula, H. Georgi, and G. S. L., p. 88 (1984), Fifth Workshop on Grand Unification, K. Kang, H. Fried, and P. Frampton eds., World Scientific, Singapore.

[101] G. Lazarides, C. Panagiotakopoulos, and Q. Shafi, Phys. Lett. B315, 325 (1993), hep-ph/9306332.

[102] G. Lazarides and C. Panagiotakopoulos, Phys. Lett. B336, 190 (1994), hep-ph/9403317.

[103] E. Ma, Phys. Rev. D36, 274 (1987).

[104] N. Irges and G. Zoupanos, Phys. Lett. B 698 (2011) 146

[105] N. Irges, G. Orfanidis and G. Zoupanos, PoS CORFU 2011 (2011) 105

[106] M. Mondragón, S. Heinemeyer, N. Tracas and G. Zoupanos, PoS CORFU2016 (2017) 041.

[107] M. Mondragón, N.D. Tracas, G. Zoupanos, Phys. Lett. B 728, 51 (2014).

[108] M. Tanabashi et al. [Particle Data Group], Phys. Rev. D 98 (2018) no.3, 030001.

[109] B. Capdevila, A. Crivellin, S. Descotes-Genon, J. Matias and J. Virto, JHEP 1801 (2018) 093 [arXiv:1704.05340 [hep-ph]].

[110] M. Misiak et al., Phys. Rev. Lett. 98 (2007) 022002 doi:10.1103/PhysRevLett.98.022002 [hep-ph/0609232]; M. Ciuchini, G. Degrassi, P. Gambino and G. F. Giudice, Nucl. Phys. B 534 (1998) 3 doi:10.1016/S0550-3213(98)00516-1 [hep-ph/9806308]; G. Degrassi, P. Gambino and G. F. Giudice, JHEP 0012 (2000) 009 doi:10.1088/1126-6708/2000/12/009 [hep-ph/0009337]; M. Carena, D. Garcia, U. Nierste and C. E. M. Wagner, Phys. Lett. B 499 (2001) 141 doi:10.1016/S0370-2693(01)00009-0 [hep-ph/0010003]; G. D’ Ambrosio, G. F. Giudice, G. Isidori and A. Strumia, Nucl. Phys. B 645 (2002) 155 doi:10.1016/S0550-3213(02)00836-2 [hep-ph/0207036].

[111] D. Asner et al. [Heavy Flavor Averaging Group], arXiv:1010.1589 [hep-ex].

[112] A. J. Buras, Phys. Lett. B 566 (2003) 115 doi:10.1016/S0370-2693(03)00561-6 [hep-ph/0303060]; G. Isidori and D. M. Straub, Eur. Phys. J. C 72 (2012) 2103 doi:10.1140/epjc/s10052-012-2103-1 [arXiv:1202.0464 [hep-ph]]. 
[113] C. Bobeth, M. Gorbahn, T. Hermann, M. Misiak, E. Stamou and M. Steinhauser, Phys. Rev. Lett. 112 (2014) 101801 doi:10.1103/PhysRevLett.112.101801 [arXiv:1311.0903 [hep-ph]]; T. Hermann, M. Misiak and M. Steinhauser, JHEP 1312 (2013) 097 doi:10.1007/JHEP12(2013)097 [arXiv:1311.1347 [hep-ph]]; C. Bobeth, M. Gorbahn and E. Stamou, Phys. Rev. D 89 (2014) no.3, 034023 doi:10.1103/PhysRevD.89.034023 [arXiv:1311.1348 [hep-ph]].

[114] R. Aaij et al. [LHCb Collaboration], Phys. Rev. Lett. 111 (2013) 101805 doi:10.1103/PhysRevLett.111.101805 [arXiv:1307.5024 [hep-ex]].

[115] S. Chatrchyan et al. [CMS Collaboration], Phys. Rev. Lett. 111 (2013) 101804 doi:10.1103/PhysRevLett.111.101804 [arXiv:1307.5025 [hep-ex]].

[116] CMS and LHCb Collaborations [CMS and LHCb Collaborations], CMS-PAS-BPH-13-007, LHCb-CONF-2013-012, CERN-LHCb-CONF-2013-012.

[117] R. Aaij et al. [LHCb Collaboration], Phys. Rev. Lett. 110 (2013) 021801 [arXiv:1211.2674 [Unknown]].

[118] G. Isidori and P. Paradisi, Phys. Lett. B 639 (2006) 499 doi:10.1016/j.physletb.2006.06.071 [hep-ph/0605012]; G. Isidori, F. Mescia, P. Paradisi and D. Temes, Phys. Rev. D 75 (2007) 115019 doi:10.1103/PhysRevD.75.115019 [hep-ph/0703035 [HEP-PH]].

[119] K. A. Olive et al. [Particle Data Group], Chin. Phys. C 38 (2014) 090001. doi:10.1088/1674-1137/38/9/090001

[120] A. J. Buras, P. Gambino, M. Gorbahn, S. Jager and L. Silvestrini, Nucl. Phys. B 592 (2001) 55 doi:10.1016/S0550-3213(00)00582-4 [hep-ph/0007313].

[121] R. Aaij et al. [LHCb Collaboration], New J. Phys. 15 (2013) 053021 doi:10.1088/1367-2630/15/5/053021 [arXiv:1304.4741 [hep-ex]].

[122] H. Goldberg, Phys. Rev. Lett. 50 (1983) 1419 Erratum: [Phys. Rev. Lett. 103 (2009) 099905]. doi:10.1103/PhysRevLett.103.099905, 10.1103/PhysRevLett.50.1419; J. R. Ellis, J. S. Hagelin, D. V. Nanopoulos, K. A. Olive and M. Srednicki, Nucl. Phys. B 238 (1984) 453. doi:10.1016/0550-3213(84)90461-9

[123] E. Komatsu et al. [WMAP Collaboration], Astrophys. J. Suppl. 192 (2011) 18 doi:10.1088/0067-0049/192/2/18 [arXiv:1001.4538 [astro-ph.CO]].

[124] E. Komatsu et al. [WMAP Science Team], PTEP 2014 (2014) 06B102 doi:10.1093/ptep/ptu083 [arXiv:1404.5415 [astro-ph.CO]].

[125] S. Heinemeyer, Int. J. Mod. Phys. A 21 (2006) 2659 doi:10.1142/S0217751X06031028 [hep-ph/0407244].

[126] S. Heinemeyer, W. Hollik and G. Weiglein, Phys. Rept. 425 (2006) 265 doi:10.1016/j.physrep.2005.12.002 [hep-ph/0412214].

[127] A. Djouadi, Phys. Rept. 459 (2008) 1 doi:10.1016/j.physrep.2007.10.005 [hep-ph/0503173].

[128] S. Heinemeyer, O. Stal and G. Weiglein, Phys. Lett. B 710 (2012) 201 doi:10.1016/j.physletb.2012.02.084 [arXiv:1112.3026 [hep-ph]].

[129] P. Bechtle, S. Heinemeyer, O. Stal, T. Stefaniak, G. Weiglein and L. Zeune, Eur. Phys. J. C 73 (2013) no.4, 2354 doi:10.1140/epjc/s10052-013-2354-5 [arXiv:1211.1955 [hep-ph]].

[130] P. Bechtle, H. E. Haber, S. Heinemeyer, O. Stal, T. Stefaniak, G. Weiglein and L. Zeune, Eur. Phys. J. C 77 (2017) no.2, 67 doi:10.1140/epjc/s10052-016-4584-9 [arXiv:1608.00638 [hep-ph]]. 
[131] G. Aad et al. [ATLAS and CMS Collaborations], Phys. Rev. Lett. 114 (2015) 191803 doi:10.1103/PhysRevLett.114.191803 [arXiv:1503.07589 [hep-ex]].

[132] G. Degrassi, S. Heinemeyer, W. Hollik, P. Slavich and G. Weiglein, Eur. Phys. J. C 28 (2003) 133 doi:10.1140/epjc/s2003-01152-2 [hep-ph/0212020].

[133] O. Buchmueller et al., Eur. Phys. J. C 74 (2014) no.3, 2809 doi:10.1140/epjc/s10052-014-2809-3 [arXiv:1312.5233 [hep-ph]].

[134] H. Bahl, S. Heinemeyer, W. Hollik and G. Weiglein, Eur. Phys. J. C 78 (2018) no.1, 57 doi:10.1140/epjc/s10052-018-5544-3 [arXiv:1706.00346 [hep-ph]].

[135] S. Heinemeyer, W. Hollik and G. Weiglein, Comput. Phys. Commun. 124 (2000) 76 doi:10.1016/S0010-4655(99)00364-1 [hep-ph/9812320]; S. Heinemeyer, W. Hollik and G. Weiglein, Eur. Phys. J. C 9 (1999) 343 doi:10.1007/s100529900006, 10.1007/s100520050537 [hep-ph/9812472]; M. Frank, T. Hahn, S. Heinemeyer, W. Hollik, H. Rzehak and G. Weiglein, JHEP 0702 (2007) 047 doi:10.1088/1126-6708/2007/02/047 [hep-ph/0611326]; T. Hahn, S. Heinemeyer, W. Hollik, H. Rzehak and G. Weiglein, Comput. Phys. Commun. 180 (2009) 1426. doi:10.1016/j.cpc.2009.02.014; T. Hahn, S. Heinemeyer, W. Hollik, H. Rzehak and G. Weiglein, Phys. Rev. Lett. 112 (2014) no.14, 141801 doi:10.1103/PhysRevLett.112.141801 [arXiv:1312.4937 [hep-ph]]; H. Bahl and W. Hollik, Eur. Phys. J. C 76 (2016) no.9, 499 doi:10.1140/epjc/s10052-016-4354-8 [arXiv:1608.01880 [hep-ph]]; See http: //www. feynhiggs.de.

[136] [ATLAS and CDF and CMS and D0 Collaborations], arXiv:1403.4427 [hep-ex].

[137] M. Carena, D. Garcia, U. Nierste and C. E. M. Wagner, Nucl. Phys. B 577 (2000) 88 doi:10.1016/S0550-3213(00)00146-2 [hep-ph/9912516].

[138] S. Heinemeyer, M. Mondragon and G. Zoupanos, Int. J. Mod. Phys. Conf. Ser. 13 (2012) 118. doi:10.1142/S2010194512006782

[139] S. Heinemeyer, M. Mondragon and G. Zoupanos, Phys. Part. Nucl. 44 (2013) 299. doi:10.1134/S1063779613020159

[140] https://twiki.cern.ch/twiki/bin/view/AtlasPublic/SupersymmetryPublicResults, https://twiki.cern.ch/twiki/bin/view/CMSPublic/PhysicsResultsSUS

[141] M. Mangano, CERN Yellow Report CERN 2017-003-M doi:10.23731/CYRM-2017-003 [arXiv:1710.06353 [hep-ph]].

[142] G. Belanger, F. Boudjema, A. Pukhov and A. Semenov, Comput. Phys. Commun. 149 (2002) 103 doi:10.1016/S0010-4655(02)00596-9 [hep-ph/0112278].

[143] G. Belanger, F. Boudjema, A. Pukhov and A. Semenov, Comput. Phys. Commun. 174 (2006) 577 doi:10.1016/j.cpc.2005.12.005 [hep-ph/0405253].

[144] D. Barducci, G. Belanger, J. Bernon, F. Boudjema, J. Da Silva, S. Kraml, U. Laa and A. Pukhov, Comput. Phys. Commun. 222 (2018) 327 doi:10.1016/j.cpc.2017.08.028 [arXiv:1606.03834 [hep-ph]].

[145] J. W. F. Valle, PoS corfu 98 (1998) 010 [hep-ph/9907222].

[146] J. W. F. Valle, PoS corfu 98 (1998) 010 [hep-ph/9907222] and references therein.

[147] M. A. Diaz, M. Hirsch, W. Porod, J. C. Romao and J. W. F. Valle, Phys. Rev. D 68 (2003) 013009 Erratum: [Phys. Rev. D 71 (2005) 059904] doi:10.1103/PhysRevD.71.059904, 10.1103/PhysRevD.68.013009 [hep-ph/0302021]. 
[148] H. K. Dreiner, Adv. Ser. Direct. High Energy Phys. 21 (2010) 565 doi:10.1142/9789814307505_0017 [hep-ph/9707435]; G. Bhattacharyya, In *Tegernsee 1997, Beyond the desert 1997* 194-201 [hep-ph/9709395]; B. C. Allanach, A. Dedes and H. K. Dreiner, Phys. Rev. D 60 (1999) 075014 doi:10.1103/PhysRevD.60.075014 [hep-ph/9906209]; J. C. Romao and J. W. F. Valle, Nucl. Phys. B 381 (1992) 87. doi:10.1016/0550-3213(92)90641-N

[149] G. Bennett et al. [The Muon g-2 Collaboration], Phys. Rev. Lett. 92, 161802 (2004), [arXiv:hep-ex/0401008]; and Phys. Rev. D 73, 072003 (2006) [arXiv:hep-ex/0602035].

[150] D. Stockinger, J. Phys. G 34 (2007) R45 [hep-ph/0609168].

[151] F. Jegerlehner and R. Szafron, Eur. Phys. J. C 71 (2011) 1632 [arXiv:1101.2872 [hep-ph]].

[152] M. Benayoun, P. David, L. DelBuono and F. Jegerlehner, Eur. Phys. J. C 73 (2013) 2453 [arXiv:1210.7184 [hep-ph]].

[153] D. H. Lyth and E. D. Stewart, Phys. Rev. D 53 (1996) 1784 doi:10.1103/PhysRevD.53.1784 [hep-ph/9510204].

[154] G. B. Gelmini and P. Gondolo, Phys. Rev. D 74 (2006) 023510 doi:10.1103/PhysRevD.74.023510 [hep-ph/0602230].

[155] S. Mandelstam, Nucl. Phys. B 213 (1983) 149. doi:10.1016/0550-3213(83)90179-7

[156] L. Brink, O. Lindgren and B. E. W. Nilsson, Phys. Lett. 123B (1983) 323. doi:10.1016/0370-2693(83)91210-8

[157] Z. Bern, J. J. Carrasco, L. J. Dixon, H. Johansson and R. Roiban, Phys. Rev. Lett. 103 (2009) 081301 doi:10.1103/PhysRevLett.103.081301 [arXiv:0905.2326 [hep-th]].

[158] R. Kallosh, JHEP 0909 (2009) 116 doi:10.1088/1126-6708/2009/09/116 [arXiv:0906.3495 [hep-th]].

[159] Z. Bern, J. J. Carrasco, L. J. Dixon, H. Johansson, D. A. Kosower and R. Roiban, Phys. Rev. Lett. 98 (2007) 161303 doi:10.1103/PhysRevLett.98.161303 [hep-th/0702112].

[160] Z. Bern, L. J. Dixon and R. Roiban, Phys. Lett. B 644 (2007) 265 doi:10.1016/j.physletb.2006.11.030 [hep-th/0611086].

[161] M. B. Green, J. G. Russo and P. Vanhove, Phys. Rev. Lett. 98 (2007) 131602 doi:10.1103/PhysRevLett.98.131602 [hep-th/0611273].

[162] J. M. Maldacena, Int. J. Theor. Phys. 38 (1999) 1113 [Adv. Theor. Math. Phys. 2 (1998) 231] doi:10.1023/A:1026654312961, 10.4310/ATMP.1998.v2.n2.a1 [hep-th/9711200].

[163] S. Heinemeyer, M. Mondragon, G. Patellis, N. Tracas and G. Zoupanos, Symmetry 10 (2018) no.3, 62 doi:10.3390/sym10030062 [arXiv:1802.04666 [hep-ph]].

[164] S. Heinemeyer, M. Mondragon, N. Tracas and G. Zoupanos, arXiv:1904.00410 [hep-ph] (in press).

[165] S. Heinemeyer, M. Mondragon and G. Zoupanos, SIGMA 6 (2010) 049 doi:10.3842/SIGMA.2010.049 [arXiv:1001.0428 [hep-ph]].

[166] S. Heinemeyer, M. Mondragon, N. Tracas and G. Zoupanos, Nucl. Phys. B 927 (2018) 319. doi:10.1016/j.nuclphysb.2017.12.010

[167] T. Kobayashi, J. Kubo, M. Mondragón and G. Zoupanos, Surveys High Energ. Phys. 16, 87 (2001).

[168] M. Mondragon and G. Zoupanos, Phys. Part. Nucl. Lett. 8 (2011) 173.

[169] Particle Data Group, C. Amsler et al., Phys. Lett. B667, 1 (2008). 
[170] S. Heinemeyer, M. Mondragón, N. Tracas and G. Zoupanos, JHEP 1808 (2018) 150.

[171] CMS Collaboration, CMS-DP-2016-064.

[172] G. Aad et al. [ATLAS and CMS Collaborations], JHEP 1608, 045 (2016) [arXiv:1606.02266 [hep-ex]]. 